\title{
Oscillation tomography of the Earth with solar neutrinos and future experiments
}

\author{
P. Bakhti $\oplus^{1}$ and A. Yu. Smirnov ${ }^{2,3}$ \\ ${ }^{1}$ School of physics, Institute for Research in Fundamental Sciences (IPM), \\ P.O. Box 19395-5531, Tehran, Iran \\ ${ }^{2}$ Max-Planck Institute for Nuclear Physics, Saupfercheckweg 1, D-69117 Heidelberg, Germany \\ ${ }^{3}$ ICTP, Strada Costiera 11, 34014 Trieste, Italy
}

(Received 31 January 2020; accepted 8 June 2020; published 29 June 2020)

\begin{abstract}
We study in detail the Earth matter effects on the boron neutrinos from the Sun using recently developed 3D models of the Earth. The models have a number of new features of the density profiles, in particular, a substantial deviation from spherical symmetry. In this connection, we further elaborate on relevant aspects of oscillations ( $\epsilon^{2}$ corrections, adiabaticity violation, entanglement, etc.) and the attenuation effect. The night excesses of the $\nu e-$ and $\nu N-$ events and the day-night asymmetries, $A_{N D}$, are presented in terms of the matter potential and the generalized energy resolution functions. The energy dependences of the cross section and the flux improve the resolution, and consequently, sensitivity to remote structures of the profiles. The nadir angle $(\eta)$ dependences of $A_{N D}$ are computed for future detectors DUNE, THEIA, HyperKamiokande, and MICA at the South pole. Perspectives of the oscillation tomography of the Earth with the boron neutrinos are discussed. Next-generation detectors will establish the integrated day-night asymmetry with high confidence level. They can give some indications of the $\eta-$ dependence of the effect, but will discriminate among different models at most at the $(1-2) \sigma$ level. For high-level discrimination, the MICA-scale experiments are needed. MICA can detect the ice-soil borders and perform unique tomography of Antarctica.
\end{abstract}

DOI: 10.1103/PhysRevD.101.123031

\section{INTRODUCTION}

Oscillations of the solar neutrinos in the Earth [1-33] have the following features.

(1) Because of loss of the propagation coherence, the solar neutrinos arrive at the surface of the Earth as independent fluxes of the mass eigenstates [8,9,15,24].

(2) Inside the Earth, the mass states oscillate in a multilayer medium with smoothly (adiabatically) changing density within layers and sharp density change at the borders between the layers.

(3) The oscillations proceed in the low-density regime, which is quantified by a small parameter

$$
\epsilon \equiv \frac{2 V E}{\Delta m_{21}^{2}}
$$

where $V(x)=\sqrt{2} G_{F} n_{e}(x)$ is the matter potential and $n_{e}$ is the electron number density of the medium.

Published by the American Physical Society under the terms of the Creative Commons Attribution 4.0 International license. Further distribution of this work must maintain attribution to the author(s) and the published article's title, journal citation, and DOI. Funded by SCOAP ${ }^{3}$.
For $E=10 \mathrm{MeV}$ at the surface of the Earth $\epsilon$ equals $\sim 0.03$.

(4) The oscillation length

$$
l_{m} \approx l_{\nu} \approx 330 \mathrm{~km}\left(\frac{E}{10 \mathrm{MeV}}\right)\left(\frac{7.5 \times 10^{-5} \mathrm{eV}^{2}}{\Delta m_{21}^{2}}\right)
$$

is comparable to a section of trajectory in a layer $d_{i}$ for trajectories with nadir angles $\eta$ close to $\pi / 2$ : $d_{i}=r_{i} / \cos \eta$, where $r_{i} \sim 10 \mathrm{~km}$ is the width of the layer in the radial direction. The highest sensitivity is to structures of the density profile of the size $\sim l_{m} / 2$.

(5) The attenuation effect is realized in the order $\epsilon$ due to the finite neutrino energy resolution (reconstruction) in the experimental setup [26,33]. It means loss of sensitivity to remote structures of the Earth density profile. Consequently, only structures sufficiently close to a detector, and therefore to the surface of the Earth (crust, upper mantle), are most relevant for observations. This means that with the boron neutrinos, deep structures, like the core of the Earth, are not seen at the $\epsilon$ level. The attenuation effect is absent in the order $\epsilon^{2}$. Thus, the solar neutrino tomography is essentially sensitive to the small scale structures in the crust and mantle of the Earth. 
In previous computations, (see, e.g., Refs. [20,24]) the density profile of the one-dimensional PREM model [34] was used. In this model, borders between layers have forms of ideal spheres. Recently, several new three dimensional Earth models have been developed. They show several new features of the density profiles which have not been taken into account previously: (i) the borders between layers are not spherically symmetric but have irregular deviations from spheres; (ii) the profiles depend on the azimuthal angle; (iii) the profiles are nonsymmetric with respect to the center of neutrino trajectory. The horizontal sizes of these structures are comparable to oscillation length, which means that effectively they can smooth borders between layers as well as produce some new parametric effects in oscillations.

In the present paper, we study how these new features modify the observational effects. We compute the Earth matter effect using new models. This allows us to assess the possibility to distinguish the models with solar neutrino detectors. At the same time, our computations quantify errors of the computed effects due to uncertainty in the density profile.

Presently, there is the first (about $3 \sigma$ ) indication of the Earth matter effect by SuperKamiokande [29], and this situation will stay until the next generation of experiments will start to operate. Here we consider solar neutrino studies by future detectors DUNE [35], Hyper-Kamiokande (HK) [36], THEIA [37,38], and MICA [39].

The paper is organized as follows. In Sec. II we present oscillation formalism relevant for our computations and elaborate on some new features, such as high order $\epsilon$ corrections, entanglement, etc. We introduce the generalized energy resolution functions and study their properties. The day-night asymmetry is presented in terms of these resolution functions and potential. In Sec. III new models of the density distribution in the Earth are described. In Sec. IV we present results of computations of the Earth matter effect for future detectors. Conclusions are given in Sec. V.

\section{RELATIVE EXCESS OF THE NIGHT EVENTS AND ATTENUATION}

\section{A. Coherence and entanglement}

Loss of the propagation coherence is due to spatial separation of the wave packets that correspond to the mass eigenstates originated from the same flavor state. Although separated, these wave packets belong to the same wave function and are therefore entangled. If one of the eigenstates is detected, the parts of the wave function which describe two other eigenstates collapse. It can be easily shown that the observational result is the same as in the case of independent fluxes of mass eigenstates once total flux of these states is normalized on the total flux of the originally produced flavor neutrinos. Coherence is not restored in a realistic detector.

\section{B. Corrections to probability}

Recall that the $\nu_{e}$ survival probability during a day, as a function of the neutrino energy, equals

$$
P_{D}(E)=\frac{1}{2} c_{13}^{4}\left[1+\cos 2 \theta_{12} \cos 2 \bar{\theta}_{12}^{m}(E)\right]+s_{13}^{4},
$$

where $c_{13} \equiv \cos \theta_{13}, \quad s_{13} \equiv \sin \theta_{13}$, and $\cos 2 \bar{\theta}_{12}^{m}$ is the mixing parameter averaged over the boron neutrino production region in the Sun [40]:

$$
\cos 2 \bar{\theta}_{12}^{m} \approx \frac{\cos 2 \theta_{12}-c_{13}^{2} \bar{\epsilon}_{\odot}}{\sqrt{\left(\cos 2 \theta_{12}-c_{13}^{2} \bar{\epsilon}_{\odot}\right)^{2}+\sin ^{2} 2 \theta_{12}}} .
$$

Here,

$$
\bar{\epsilon}_{\odot} \equiv \frac{2 \bar{V}_{\odot} E}{\Delta m_{21}^{2}}
$$

and $\bar{V}_{\odot}$ is the averaged matter potential in the ${ }^{8} \mathrm{~B}$ neutrino production region.

For the high energy part of the boron neutrino spectrum, where $\bar{\epsilon}_{\odot} \gg 1$, we have

$$
\cos 2 \theta_{12}^{m}(E) \approx-\left[1-\frac{\sin ^{2} 2 \theta_{12}}{2 c_{13}^{4}}\left(\frac{\Delta m_{21}^{2}}{2 \bar{V}_{\odot} E}\right)\right] .
$$

So, dependence on $E$ is weak. At the solar neutrino energies the matter effect on the 1-3 mixing is negligible, therefore $\bar{\theta}_{13} \approx \theta_{13}=8.4^{\circ}[41]$.

During a night the probability equals $P_{N}=P_{D}+\Delta P$, where the difference of the night and day probabilities is given to the order $\epsilon^{2}$ by $[26,42]$

$\Delta P(E)=\kappa(E)\left[\int_{0}^{L} d x V(x) \sin \phi^{m}(L-x, E)+I_{2}\right]$.

Here

$$
\kappa(E) \equiv-\frac{1}{2} c_{13}^{6} \cos 2 \bar{\theta}_{12}^{\odot}(E) \sin ^{2} 2 \theta_{12} \approx 0.5
$$

is slowly changing function of $E$, and

$$
I_{2} \equiv \frac{1}{2} \cos 2 \theta_{12}\left[\int_{0}^{L} d x V(x) \cos \phi^{m}(L-x)\right]^{2}
$$

is a correction of the order $\epsilon^{2}$, since in Eq. (7) each integral over $x$ is of the order $\epsilon$. The integration in Eq. (7) proceeds along a neutrino trajectory. In new models of the Earth apart from the nadir angle $\eta$ the density and potential profiles depend also on position of the detector $\mathbf{x}_{D}$ and azimuthal angle $\phi_{a}: V=V\left(x, \mathbf{x}_{D}, \eta, \phi_{a}\right)$. Correspondingly, for a given detector and a given moment of time, $\Delta P=\Delta P\left(\mathbf{x}_{D}, \eta, \phi_{a}\right)$. 
In Eq. (6)

$$
\phi^{m}(L-x, E) \equiv \int_{x}^{L} d x \Delta_{21}^{m}(x)
$$

is the adiabatic phase acquired from a given point of trajectory $x$ to a detector at $L . \Delta_{21}^{m}(x)$ is the level splitting and in our calculations we use it up to the first order in $\epsilon$ :

$$
\begin{aligned}
\Delta_{21}^{m} & =\Delta_{21} \sqrt{\left(\cos 2 \theta_{21}-c_{13}^{2} \epsilon\right)^{2}+\sin ^{2} 2 \theta_{21}} \\
& \approx \Delta_{21}\left(1-c_{13}^{2} \cos 2 \theta_{12} \epsilon\right) .
\end{aligned}
$$

Here $\Delta_{21} \equiv \Delta m_{21}^{2} / 2 E$ is the splitting in vacuum. Consequently, the oscillation phase (8) equals

$\phi^{m}(L-x, E)=\Delta_{21}\left[(L-x)-c_{13}^{2} \cos 2 \theta_{12} \int_{x}^{L} d x \epsilon(x)\right]$.

Introducing the average density along a neutrino trajectory $\bar{\rho}(\eta)$, we can rewrite Eq. (9) as

$$
\phi^{m}(L-x, E)=\phi_{0}^{m}+\delta \phi^{m},
$$

where $\phi_{0}^{m} \equiv \Delta_{21}(L-x)$ is the zero order phase and

$$
\delta \phi^{m}=\phi_{0}^{m} c_{13}^{2} \cos 2 \theta_{12} \epsilon(\bar{\rho})
$$

is the phase shift due to the $\epsilon-$ correction.

For $\Delta m^{2}=7.5 \times 10^{-5} \mathrm{eV}^{2}$ and $\rho=5 \mathrm{~g} / \mathrm{cm}^{3}$ the relative size of the correction [second term in Eq. (10)] is about $3 \%$. For large $\phi_{0}^{m}$ the phase shift $\delta \phi^{m}$ can be observable. E.g., if $\phi_{0}^{m}=5 \pi$, we find $\delta \phi^{m}=27^{\circ}$.

The correction $\delta \phi^{m}$ leads to the shift of oscillatory pattern in the $\eta$ scale. Since $\delta \phi^{m}=\Delta_{21} \delta L(\eta)$ and $L=$ $2 R \cos \eta$ we obtain

$$
\delta \eta=\frac{\delta \phi^{m}}{2 R \sin \eta \Delta_{21}} .
$$

Insertion of expression for $\delta \phi^{m}$ (11) into Eq. (12) gives

$$
\delta \eta=\cot \eta c_{13}^{2} \cos 2 \theta_{12} \epsilon .
$$

For $\eta=70^{\circ}$ we obtain $\delta \eta=0.2^{\circ}$, while the period of oscillatory dependence in the $\eta$ scale for this $\eta$ equals $2.8^{\circ}$, i.e., the shift is by $1 / 14$ of the period. $\delta \eta$ increases with decrease of $\eta$.

Let us consider $I_{2}$ - the second term in Eq. (6). For constant density it can be computed explicitly

$$
I_{2} \approx 0.5 \cos 2 \theta_{12} \bar{\epsilon}^{2} \sin ^{2}\left(L \Delta_{21}\right) .
$$

Apart from $\bar{\epsilon}^{2}$, this term contains an additional small factor $0.5 \cos 2 \theta_{12} \approx 1 / 6$. As a result, $I_{2}$ is about $0.015 \%$ and therefore can be neglected. Our computational relative errors are of the order of $0.1 \%$. Thus, the largest correction to the probability follows from $\phi^{m}$.

\section{Comments on adiabaticity}

In the lowest order in $\epsilon$, the sensitivity to structures of the Earth matter profile, its deviation from constant density, appears due to borders between layers which strongly (maximally) break adiabaticity. Indeed, in the adiabatic case the oscillation probability would depend on density at the surface of the Earth and on the oscillation phase. However, in the lowest (zero) order in $\epsilon$ the phase coincides with the vacuum phase. The matter correction to the phase is proportional to $\epsilon$ which then appears as $\epsilon^{2}$ in the probability. So, in the adiabatic case, there is no sensitivity to the profile in the $\epsilon$ order.

In general, deviations of borders between layers from the spherical form may produce effective smearing of borders for neutrino trajectories with large $\eta$, and consequently, to a decrease of the adiabaticity violation. That would lead to partial loss of sensitivity to the density profile.

If deviation from the spherical form in the radial direction $\Delta h$ and in the horizontal direction $l_{f}$ are such that neutrino trajectory at certain $\eta$ crosses the border between the same layers several (many) times, the density gradient along the trajectory will decrease. For density jump in a border $\Delta \rho$ the gradient equals $\Delta \rho \cos \eta / \Delta h$. The scale of density change

$$
l_{\rho} \equiv \rho(d \rho / d l)^{-1}=\frac{\rho}{\cos \eta \Delta \rho} \Delta h
$$

should be compared with the oscillation length in the adiabaticity condition.

As we will see, the typical scale of deviation of, e.g., the border between the crust and mantle from the spherical form is $\Delta h \sim 5-10 \mathrm{~km}$ and the horizontal size of the structures is $l_{f} \sim(70-150) \mathrm{km}$. This gives the slope of the structure $\eta_{f} \sim \Delta h / l_{f} \sim(2-7)^{\circ}$. Therefore, double crossing can occur for the trajectories with $\eta>83^{\circ}$. For parameters of new Earth models, however, adiabaticity is still strongly broken and multiple crossing of borders can occur only in very narrow intervals of $\eta$.

In the lowest $\epsilon$ order, the result for $\Delta P(E)$ in Eq. (6) can be reproduced as a result of interference of the "oscillation waves" emitted from borders between layers [42]. For $i$ th wave, the phase is determined by distance from border to a detector $L-x_{i}$ and vacuum oscillation length, while the amplitude is proportional by the density jump $\Delta \rho_{i}$ in the border. Then $\Delta P(E)$ is the sum of the waves over borders which neutrino trajectory crosses. This representation gives simple interpretation of results of numerical computations. 


\section{Attenuation and generalized energy resolution functions}

The Earth matter effect can be quantified by the day-night asymmetry or the relative excess of night events (events rate) in energy range $\Delta E$ as a function of the nadir angle $\eta$ :

$A_{N D}(\eta, \Delta E) \equiv \frac{\Delta N_{N}(\eta, \Delta E)}{N_{D}(\Delta E)}, \quad \Delta N_{N} \equiv N_{N}-N_{D}$.

Here $N_{N}(\eta)$ and $N_{D}$ are the numbers of night and day events (rates) correspondingly. The nadir $\eta$ and azimuthal $\phi_{a}$ angles are fixed by the detection time of an event. According to new models, $N_{N}(\eta)$ depends also on the position of a detector.

In experiments, the observables are the electron energy and direction. Therefore, $\Delta E$ is determined by the observed energy interval of the produced (or recoil) electrons. In practice, we will use the energy of electrons above a certain threshold. Thus, information on the density profile is encoded in the nadir angle dependence of the night excess. We will not consider the direction of the electron.

Sensitivity of oscillations to the Earth density profile is determined by the sensitivity of a given experimental setup to the true energy of neutrino $E$. This can be described by the generalized energy resolution function $G_{\nu}\left(E^{r}, E\right)$ such that

$$
\Delta N\left(E^{r}\right)=D \int d E G_{\nu}\left(E^{r}, E\right) \Delta P(E)
$$

where $E^{r}$ is the observed (reconstructed) neutrino energy or certain energy characteristics which can be measured in experiment. In Eq. (16) $D$ is the factor that includes characteristics of detection: fiducial volume, exposure time, etc. It cancels in the expression for the relative excess $A_{N D}$. The resolution function is normalized as $\int G_{\nu}\left(E^{r}, E\right) d E=1$. Similarly, one can write an expression for $N_{D}$.

$G_{\nu}\left(E^{r}, E\right)$ includes the neutrino energy resolution function: $g_{\nu}\left(E^{r}, E\right)$, the energy dependence of the neutrino flux $f_{B}(E)[40]$ and cross section $\sigma(E)$ :

$$
G_{\nu}\left(E^{r}, E\right) \propto g_{\nu}\left(E^{r}, E\right) \sigma(E) f_{B}(E) .
$$

It should also include the energy dependent efficiency of detection.

Integration over the neutrino energy with the resolution function in Eq. (16) leads to the attenuation effect [24,33]. Plugging the expression for $\Delta P(E)$ from Eq. (6) into Eq. (16) and neglecting $I_{2}$ we obtain for $\Delta N$

$D \int_{0}^{L} d x V(x) \int_{0}^{E^{\max }} d E G_{\nu}\left(E^{r}, E\right) \sin \phi^{m}(L-x, E)$.

Here integrations over $x$ and $E$ are interchanged. In this form the dependence of difference of events on structures of density profile is immediate.
Let us introduce the attenuation factor $F(L-x)$ [24] such that the integral over $E$ in Eq. (18) equals

$$
\begin{array}{r}
\int d E G_{\nu}\left(E^{r}, E\right) \sin \phi^{m}(L-x, E) \\
=F(L-x) \sin \phi^{m}\left(L-x, E^{r}\right) .
\end{array}
$$

In general, this equality cannot be satisfied, but it is valid for special cases and under integral over $x$. Then the expression for $\Delta N$ in Eq. (18) becomes

$\Delta N\left(E^{r}\right)=D \int d x V(x) F(L-x) \sin \phi^{m}\left(L-x, E^{r}\right)$.

For the Gaussian form of $G_{\nu}\left(E^{r}, E\right)$, the attenuation factor is given by

$$
F(d) \simeq e^{-2\left(\frac{d}{\lambda_{\text {att }}}\right)^{2}},
$$

where

$$
\lambda_{\text {att }} \equiv l_{\nu} \frac{E}{\pi \sigma_{E}}
$$

is the attenuation length, and $l_{\nu}$ is the oscillation length in vacuum

$$
l_{\nu}=\frac{4 \pi E}{\Delta m_{21}^{2}} .
$$

According to Eqs. (20) and (21) for $d \gg \lambda_{\text {att }}$ the attenuation factor $F(d) \approx 0$, and therefore contributions of remote structures to the integral (20) and therefore to observable oscillation effect is suppressed. For $d=\lambda_{\text {att }}$ the factor $F(d)=e^{-2} \approx 0.14$, and the attenuation becomes significant. Consequently, the day-night asymmetry depends mainly on the shallow structures of the Earth which are close to a detector.

For the ideal resolution, $G_{\nu}\left(E^{r}, E\right)=\delta\left(E^{r}-E\right)$, Eq. (19) gives $F(L-x)=1$, which means that attenuation is absent.

The attenuation length is the distance at which oscillations integrated over the energy resolution interval $\sigma_{E}$ are averaged out, or the difference of the oscillation phases for $E$ and $E+\sigma_{E}$ becomes larger than $2 \pi$ [33].

Expression (18) factorizes different dependences: The generalized resolution function encodes external characteristics: neutrino flux, cross section, energy resolution of a detector. $V(x)$ gives information about the density profile, oscillation probability is reduced to $\sin \phi^{m}$.

In what follows we will find expressions for the generalized reconstruction functions and present numbers of events in the form (18) separately for the $\nu$ - nucleon and $\nu-e$ scattering. 


\section{E. Neutrino-nuclei scattering}

We consider the charged current neutrino-nuclei interactions and the corresponding resolution function $G_{\nu N}$. If transitions to excited states are neglected, the energies of electron and neutrino are uniquely related (up to negligible nuclei recoil): $E_{e}=E-\Delta E$. Here $\Delta E \approx \Delta M+m_{e}$ is the threshold of reaction. If transitions to excited states are significant but the energy of deexcitation is not measured, an additional uncertainty in reconstruction of the neutrino energy appears which should be included into $G_{\nu N}$.

The night-day difference of numbers of events with the observed energy of electron $E_{e}^{r}$ is given by

$$
\Delta N\left(E_{e}^{r}\right)=D \int_{0}^{E_{e}^{\max }} d E_{e} g_{e}\left(E_{e}^{r}, E_{e}\right) \sigma(E) f_{B}(E) \Delta P(E),
$$

where $E=E_{e}+\Delta E, E_{e}^{\max }$ is maximal true energy of electron: $E_{e}^{\max }=E^{\max }-\Delta E, g_{e}\left(E_{e}^{r}, E_{e}\right)$ is the electron energy resolution function with $E_{e}$ and $E_{e}^{r}$ being the true and the observed energies correspondingly.

Introducing also $E^{r} \equiv E_{e}^{r}+\Delta E$ and changing integration in Eq. (24) to integration over the neutrino energy $E$ we have

$$
\Delta N\left(E_{e}^{r}\right)=D \int_{\Delta E}^{E^{\max }} d E g_{\nu}\left(E^{r}, E\right) \sigma(E) f_{B}(E) \Delta P(E),
$$

where $g_{\nu}\left(E^{r}, E\right) \equiv g_{e}\left(E^{r}-\Delta E, E-\Delta E\right)$. Equation (25) can be rewritten as

$$
\Delta N\left(E_{e}^{r}\right)=D z \sigma\left(E^{r}\right) f_{B}\left(E^{r}\right) \int_{0}^{E^{\max }} d E G_{\nu N}\left(E^{r}, E\right) \Delta P(E)
$$

with

$$
G_{\nu N}\left(E^{r}, E\right)=z^{-1} g_{\nu}\left(E^{r}, E\right) \frac{\sigma(E) f_{B}(E)}{\sigma\left(E^{r}\right) f_{B}\left(E^{r}\right)},
$$

and $z$ being the normalization factor. Inserting the expression for $\Delta P(E)$ from Eq. (6) into Eq. (26) and permuting integrations over $x$ and $E$ we obtain

$$
\begin{aligned}
\Delta N\left(E_{e}^{r}\right)= & D z \sigma\left(E^{r}\right) f_{B}\left(E^{r}\right) \kappa\left(E^{r}\right) \\
& \times \int d x V(x) \int_{0}^{E_{\nu}^{\max }} d E G_{\nu N}\left(E^{r}, E\right) \sin \phi^{m}(x, E),
\end{aligned}
$$

Integration over the energy can be removed, introducing of the attenuation factor, as in Eq. (19), which gives

$$
\begin{aligned}
\Delta N\left(E_{e}^{r}\right)= & D z \sigma\left(E^{r}\right) f_{B}\left(E^{r}\right) \kappa\left(E^{r}\right) \\
& \times \int d x V(x) F_{\nu N}(L-x) \sin \phi^{m}\left(x, E^{r}\right) .
\end{aligned}
$$

Finally, integration over the interval of observed energies of electrons gives

$$
\begin{aligned}
\Delta N\left(\Delta E_{e}^{r}\right)= & D z \int_{E^{\text {th }}}^{E^{\max }} d E^{r} \sigma\left(E^{r}\right) f_{B}\left(E^{r}\right) \kappa\left(E^{r}\right) \\
& \times \int d x V(x) F_{\nu N}(L-x) \sin \phi^{m}\left(x, E^{r}\right),
\end{aligned}
$$

where we again substituted integration over $E_{e}$ by integration over $E$.

For the day signal, which does not depend practically on $\eta$, we have

$$
\begin{aligned}
N_{D}\left(\Delta E_{e}^{r}\right)= & D z \int_{E^{\min }}^{E_{\max }} d E^{r} \sigma\left(E^{r}\right) f_{B}\left(E^{r}\right) P_{D}\left(E^{r}\right) \\
& \times \int_{\Delta E}^{E^{\max }} d E G_{\nu N}\left(E^{r}, E\right) .
\end{aligned}
$$

Notice that if threshold $\Delta E$ is low enough, the second integral over the resolution function is $\approx 1$, so that

$$
N_{D}\left(\Delta E_{e}^{r}\right)=D z \int_{E^{\min }}^{E^{\max }} d E^{r} \sigma\left(E^{r}\right) f_{B}\left(E^{r}\right) P_{D}\left(E^{r}\right) .
$$

The factors $D z$ cancel in the expression for $A_{N D}$.

Let us consider the generalized energy resolution function $G_{\nu N}\left(E^{r}, E\right)$ in detail. In the expression for $G_{\nu N}\left(E^{r}, E\right)$ in Eq. (27), we use (i) $\sigma \propto E p$, (ii) the Gaussian function for $g_{\nu}\left(E^{r}, E\right)$ with central energy $E^{c}=E^{r}$ and the energy resolution $\sigma_{E}=0.07 E_{r}$ (as for DUNE), (iii) the flux of Boron neutrinos, $f_{B}(E)$ from Ref. [40]. Figure 1 (upper panel) shows by solid lines the dependence of $G_{\nu N}$ on energy $E$ for several values of $E^{r}$. We compare this dependence with Gaussian form $g^{\text {Gauss }}\left(E^{r}, E\right)$ (dashed lines) computed with the same $E^{r}$ and $\sigma_{E}$. For convenience of comparison, we normalized $G_{\nu N}\left(E^{r}, E\right)$ in such a way that $G_{\nu N}\left(E^{r}, E\right)^{\max }=g^{\text {Gauss }}\left(E^{r}, E\right)^{\max }$; and the $y$ axis is in arbitrary units.

The figure illustrates the effect of inclusion of energy dependence of $\sigma(E)$ and $f_{B}(E)$ into the resolution function. The product $\sigma(E) f_{B}(E)$ has the form of a wide asymmetric peak with maximum at $\sim 11 \mathrm{MeV}$. Consequently, for $E^{r}=$ $11 \mathrm{MeV}$ the generalized function $G_{\nu N}$ is close to the corresponding Gaussian form with energy of maximum $E^{c} \approx E^{r}$, while for $E^{r}>11 \mathrm{MeV}$ the factor $\sigma(E) f_{B}(E)$ shifts $G_{\nu N}$ to lower energies, $E^{c}<E^{r}$, and reduces the width. According to Fig. 1 for $E^{r}=12 \mathrm{MeV}$ the energy of maximum $E^{c}=11.7 \mathrm{MeV}$ and the relative width $\sigma_{E} / E=$ 0.06 instead of 0.07 in $g_{\nu}^{\text {Gauss }}$. The change becomes more profound with increase of $E^{r}$. For $E^{r}=14 \mathrm{MeV}$ we find 

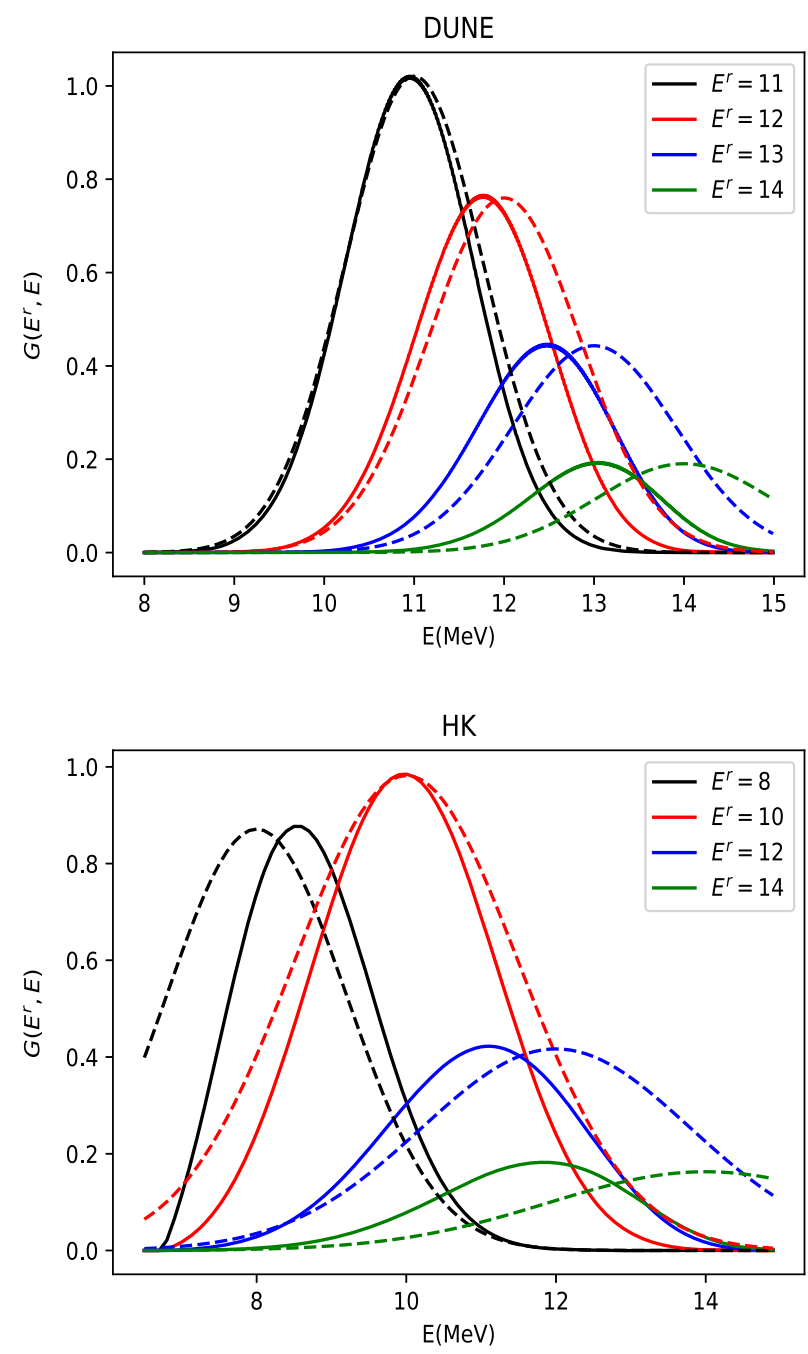

FIG. 1. Dependences of the generalized neutrino energy resolution functions $G_{\nu}\left(E^{r}, E\right)=g_{\nu}\left(E^{r}, E\right) \sigma(E) f_{B}(E)$ (solid lines) and $G_{\nu}\left(E^{r}, E\right)=g^{\text {Gauss }}\left(E^{r}, E\right)$ (dashed lines) on true neutrino energy $E$ for different values of the reconstructed neutrino energy $E^{r}$. For $g_{\nu}\left(E^{r}, E\right)$ we take the Gaussian distribution with width $\sigma_{E}$. Upper panel: $G_{\nu N}$-for experiments based on the $\nu_{e}-$ nuclei scattering with $\sigma_{E}=0.07 E^{r}$ (DUNE). Bottom panel: $G_{\nu e}$-for experiments based on the $\nu-e$ scattering with $\sigma_{E}=0.15 E^{r}$ (HK) and the cut $E_{e}>6.5 \mathrm{MeV}$.

$E^{c}=13.1 \mathrm{MeV}$ and $\sigma_{E} / E=0.05$. Thus, the energy dependence of $\sigma f_{B}$ leads to better energy resolution and therefore to increases in the attenuation length, which means the improvement of sensitivity to remote structures.

Notice that inclusion of $\sigma f_{B}$ into $G_{\nu N}$, not only gives a shift of the peak and decrease of width, but also changes the shape of the resolution function which becomes asymmetric. Still, according to Fig. 1, for Gaussian $g_{\nu}$, the whole resolution function $G_{\nu N}$ can be well approximated by the Gaussian function with appropriately chosen energy of maximum, $E^{c}=E^{c}\left(E_{r}\right) \neq E_{r}$, and width $\sigma_{E}=\sigma\left(E^{r}\right)$. A priori, the form of $g_{\nu}\left(E^{r}, E\right)$ is not known, and eventually will be determined in experiment. Therefore in our computations we will use the generalized reconstruction function in the Gaussian form:

$$
G_{\nu N}\left(E^{r}, E\right) \approx g^{\text {Gauss }}\left[E, E^{\max }\left(E^{r}\right), \sigma\left(E^{r}\right)\right] .
$$

Under integration over the neutrino energy $E$ the difference of results for $A_{N D}$ computed with the Gaussian $G_{\nu N}$ (33) and $G_{\nu N}$ with Gaussian $g_{\nu}$ is negligible. Using the PREM model we find that the relative difference result for $A_{N D}$ is smaller than $0.3 \%$.

\section{F. Neutrino-electron scattering}

In this case the energies of the neutrino and electron are not uniquely related, but correlated via the differential cross section $d \sigma\left(E, E_{e}\right) / d E_{e}$. Correspondingly, expression for the effective resolution function in Eq. (18) will differ from $G_{\nu N}$.

The difference of numbers of the night and day events with a given observed energy of electron $E_{e}^{r}$ equals

$$
\begin{aligned}
\Delta N\left(E_{e}^{r}\right)= & D \int_{0}^{E^{\max }} d E_{e} g_{e}\left(E_{e}^{r}, E_{e}\right) \\
& \times \int_{E_{e}}^{E^{\max }} d E \frac{d \sigma_{\Delta}\left(E, E_{e}\right)}{d E_{e}} \Delta P(E) f_{B}(E),
\end{aligned}
$$

where

$$
\frac{d \sigma_{\Delta}\left(E, E_{e}\right)}{d E_{e}} \equiv \frac{d \sigma_{e}\left(E, E_{e}\right)}{d E_{e}}-\frac{d \sigma_{\mu}\left(E, E_{e}\right)}{d E_{e}}
$$

is the difference of the $\nu_{e} e, d \sigma_{e} / d E_{e}$, and $\nu_{\mu} e, d \sigma_{\mu} / d E_{e}$, differential cross sections. Interchanging integrations over $E_{e}$ and $E$ in Eq. (34) we obtain

$\Delta N\left(E_{e}^{r}\right)=D \int_{0}^{E^{\max }} d E \Delta P(E) f_{B}(E) \sigma_{\Delta}(E) g_{\nu}\left(E_{e}^{r}, E\right)$,

where

$g_{\nu}\left(E_{e}^{r}, E\right) \equiv \frac{1}{\sigma_{\Delta}(E)} \int_{0}^{E} d E_{e} \frac{d \sigma_{\Delta}\left(E, E_{e}\right)}{d E_{e}} g_{e}\left(E_{e}^{r}, E_{e}\right)$,

and

$$
\sigma_{\Delta}(E)=\int_{0}^{E} d E_{e} \frac{d \sigma_{\Delta}\left(E, E_{e}\right)}{d E_{e}} .
$$

The generalized reconstruction function can be introduced similarly to Eq. (27):

$$
G_{\nu e}\left(E_{e}^{r}, E\right)=z^{-1} g_{\nu}\left(E, E_{e}^{r}\right) \frac{f_{B}(E) \sigma_{\Delta}(E)}{f_{B}\left(E_{e}^{r}\right) \sigma_{\Delta}\left(E_{e}^{r}\right)},
$$

or explicitly, inserting $g_{\nu}$ from Eq. (37), as 


$$
\begin{aligned}
G_{\nu e}\left(E_{e}^{r}, E\right)= & \frac{z^{-1} f_{B}(E)}{f_{B}\left(E_{e}^{r}\right) \sigma_{\Delta}\left(E_{e}^{r}\right)} \\
& \times \int_{0}^{E} d E_{e} \frac{d \sigma_{\Delta}\left(E, E_{e}\right)}{d E_{e}} g_{e}\left(E_{e}, E_{e}^{r}\right) .
\end{aligned}
$$

The only difference from Eq. (27) is that here in $g_{\nu}$ the electron resolution function is integrated with the differential cross section.

Instead of $E_{e}^{r}$ we can introduce the "observable" neutrino energy $E^{r}=E^{r}\left(E_{e}^{r}\right)$ defined as the energy of maximum of $G_{\nu e}$ for a given $E_{e}^{r}$ :

$$
G_{\nu e}\left(E_{e}^{r}, E^{r}\right)=G_{\nu e}^{\max }\left(E_{e}^{r}\right) .
$$

In terms of $G_{\nu e}\left(E_{e}^{r}, E\right)$ the ND difference of numbers of events can be presented as

$$
\begin{aligned}
\Delta N\left(E_{e}^{r}\right)= & D z f_{B}\left(E^{r}\left(E_{e}^{r}\right)\right) \sigma_{\Delta}\left(E^{r}\left(E_{e}^{r}\right)\right) \\
& \times \int_{0}^{E^{\max }} d E \Delta P(E) G_{\nu e}\left(E^{r}\left(E_{e}^{r}\right), E\right) .
\end{aligned}
$$

As in the $\nu N-$ case, we insert explicit expression for $\Delta P(E)$ and interchange integration over $x$ and $E$. Then the integration over $E$ can be removed introducing the attenuation factor which gives

$$
\begin{aligned}
\Delta N\left(E_{e}^{r}\right)= & D z f_{B}\left(E^{r}\right) \sigma_{\Delta}\left(E^{r}\right) \kappa\left(E^{r}\right) \\
& \times \int d x V(x) F_{\nu e}(L-x) \sin \phi^{m}\left(x, E^{r}\right),
\end{aligned}
$$

where $F_{\nu e}(L-x)$ corresponds to $G_{\nu e}\left(E^{r}, E\right)$.

The difference of numbers of events with the observable energy of electrons in the interval $\Delta E_{e}^{r} \equiv\left(E_{e}^{r, \min }-E_{e}^{r, \max }\right)$ equals

$$
\begin{aligned}
\Delta N\left(\Delta E_{e}\right)= & D z \int_{E_{e}^{r, \min }}^{E^{r, \max }} d E_{e}^{r} f_{B}\left(E^{r}\right) \sigma_{\Delta}\left(E^{r}\right) \kappa\left(E^{r}\right) \\
& \times \int d x V(x) F_{\nu e}(L-x) \sin \phi^{m}\left(x, E^{r}\right),
\end{aligned}
$$

and $E^{r}=E^{r}\left(E_{e}^{r}\right)$ is determined by Eq. (41).

The number (rate) of events with the observed electron energy $E_{e}^{r}$ during a day equals

$$
\begin{aligned}
N_{D}\left(E_{e}^{r}\right)= & \int_{0}^{E^{\max }} d E f_{B}(E)\left[P_{D}(E) \sigma^{e}\left(E, E_{e}^{\mathrm{th}}\right) g_{\nu}^{e}\left(E_{e}^{r}, E\right)\right. \\
& \left.+\left(1-P_{D}(E)\right) \sigma^{\mu}\left(E, E_{e}^{\mathrm{th}}\right) g_{\nu}^{\mu}\left(E_{e}^{r}, E\right)\right]
\end{aligned}
$$

Here

$$
g_{\nu}^{e, \mu}\left(E_{e}^{r}, E\right) \equiv \int_{0}^{E} d E_{e} \frac{d \sigma_{e, \mu}\left(E, E_{e}\right)}{\sigma_{e}(E) d E_{e}} g_{e}\left(E_{e}^{r}, E_{e}\right) .
$$

The total cross sections are given by

$$
\sigma_{e, \mu}(E)=\int_{0}^{E} d E_{e} \frac{d \sigma_{e, \mu}\left(E, E_{e}\right)}{d E_{e}} .
$$

Expression (45) can be simplified assuming $g_{\nu}^{\mu} \approx g_{\nu}^{e} \approx g_{\nu}$ :

$$
\begin{aligned}
N_{D}\left(E_{e}^{r}\right)= & \int_{0}^{E^{\max }} d E f_{B}(E) g_{\nu}\left(E_{e}^{r}, E\right)\left[P_{D}(E) \sigma^{e}\left(E, E_{e}^{\mathrm{th}}\right)\right. \\
& \left.+\left(1-P_{D}(E)\right) \sigma^{\mu}\left(E, E_{e}^{\mathrm{th}}\right)\right] .
\end{aligned}
$$

Let us consider $G_{\nu e}\left(E, E_{e}^{r}\right)$ in detail. In the bottom panel of Fig. 1 we show $G_{\nu e}\left(E, E_{e}^{r}\right)$ as a function of $E$ computed according to Eq. (40). We take the Gaussian form for $g_{\nu}\left(E^{r}, E\right)$ with central energy $E^{c}=E^{r}$ and the energy resolution $\sigma_{E}=0.15 E_{r}$. For the $\nu-e$ scattering the product $\sigma(E) f_{B}(E)$ has a wide peak with maximum at $E=10 \mathrm{MeV}$, and additional weak $E$ dependence comes from the integral in Eq. (40). Therefore the smallest deviation of $G_{\nu e}\left(E^{r}, E\right)$ from the Gaussian form is at $E^{r} \sim 10 \mathrm{MeV}$. For $E^{r}<10 \mathrm{MeV}$ the maximum of $G_{\nu e}$ is shifted to higher energies, while for $E^{r}>10 \mathrm{MeV}$, to lower energies. In both cases the width of $G_{\nu e}$ decreases. According to Fig. 1 (bottom) for $E^{r}=8 \mathrm{MeV}$ the maximum of $G_{\nu e}$ is shifted with respect to $E^{r}$ to higher energy by $0.5 \mathrm{MeV}$, and the width is slightly smaller. For $E^{r}=12 \mathrm{MeV}$, inversely, the maximum is shifted to $E^{c}=11.3 \mathrm{MeV}$, and the width becomes $\sigma_{E} / E=0.12$. This trend (due to fast decrease of the flux with energy above $10-11 \mathrm{MeV}$ ) is even more significant for larger $E^{r}$ : at $E^{r}=14 \mathrm{MeV}$, we find $E^{c}=11.9 \mathrm{MeV}$ and $\sigma_{E} / E=0.11$. Again, taking into account the energy dependence of $\sigma$ and $f_{B}$ improves the energy resolution, but this improvement is weaker than in the $\nu N$ case.

The biggest contribution to the oscillation effect comes from the energy range $(10-12) \mathrm{MeV}$, where $G_{\nu e}$ is rather close to the Gaussian form. Therefore in computations, we will use the Gaussian form for $G_{\nu e}$ with modified $E^{c}$ and $\sigma_{E}$, and, consequently, the attenuation factor in the form of Eq. (21). Inclusion of the flux and cross-section energy dependences narrows the resolution function.

In expressions for $\Delta N$ the $\phi_{a}$ dependence appears in two places: in the potential $V=V\left(x, \eta, \phi_{a}\right)$ and in the phase $\phi^{m}=\phi^{m}\left(\phi_{a}\right)$. For each $\eta$ and position of the detector we performed averaging of $\Delta N$ over the azimuthal angle $\phi_{a}$. If $\phi_{a}$ dependence of the phase is neglected, in the first approximation the averaging of $\Delta N$ over $\phi_{a}$ is reduced to averaging of the potential.

\section{MODELS OF THE EARTH AND DENSITY PROFILES}

In computations, we used density profiles reconstructed from recently developed 3D models of the Earth. Because 
of the attenuation effect, the day-night asymmetry mainly depends on shallow density structures: crust, upper mantle, and crust-mantle border called Moho, or Mohorovicic discontinuity. There are two types of crust: the oceanic crust and the continental one. The width of the oceanic crust is about $(5-10) \mathrm{km}$, while the continental crust is thicker: $(20-90) \mathrm{km}$ $[43,44]$. The predicted depth of Moho, $h_{\text {Moho }}$, significantly varies for different models. In contrast, the density change in the Moho is nearly the same for all the models. Beneath Homestake the jump is from 2.9 to $3.3 \mathrm{~g} / \mathrm{cm}^{3}$.

A brief description of relevant elements of the models is given below.

(1) The Shen-Ritzwoller model (S-R) [45] is based on joint Bayesian Monte Carlo inversion of geophysical data. It gives the density profile of the crust and uppermost mantle beneath the U.S., in area with latitudes $\left(20^{\circ}-50^{\circ}\right)$ and longitudes $\left(235^{\circ}-295^{\circ}\right)$. In the radial direction it provides the density change from the sea level surface down to the depth of $150 \mathrm{~km}$ with $h_{\text {Moho }}=52 \mathrm{~km}$ beneath the Homestake (see Fig. 2).

(2) FWEA18, the full waveform inversion of the East Asia model [46], covers the latitudes $10^{\circ}-60^{\circ}$ and longitudes $90^{\circ}-150^{\circ}$. It gives the density profile from the surface down to $800 \mathrm{~km}$, and $h_{\text {Moho }}=33 \mathrm{~km}$ beneath Kamioka.

(3) SAW642AN [47] is a global (all latitudes and longitudes) radially anisotropic mantle shear velocity model based on a global three-dimensional tomography of the Earth. The model gives the density profile of mantle starting from the depth of Moho, $h_{\text {Moho }}=24 \mathrm{~km}$, down to $2900 \mathrm{~km}$. No crust structure is available.

(4) CRUST1 [48] is a global 3D model, that presents data with $1 \times 1$ degree grid in latitude and longitude at the surface. It gives the density and depth of borders of eight layers of the crust: water, ice, upper sediments, middle sediments, lower sediments, upper crust, middle crust, and lower crust. The model predicts the depths of Moho $h_{\text {Moho }}=48$ and $h_{\text {Moho }}=40 \mathrm{~km}$ beneath Homestake and Kamioka, respectively, and nearly constant density of the upper mantle down to $100 \mathrm{~km}$. It provides also the density distribution above the sea level.

Using these models we reconstructed the density, and, consequently, $V(x)$ profiles along neutrino trajectories determined by position of detectors, $\eta$ and $\phi_{a}$. Maximal depths $h^{\text {max }}$ down to which the models provide data are $h^{\max }(\mathrm{S}-\mathrm{R})=150, \quad h^{\max }($ CRUST1 $) \approx 80$, $h^{\max }($ FWEA18 $)=800, \quad h^{\max }($ SAW642AN $)=2900 \mathrm{~km}$. Therefore, we reconstructed the density profiles using the following prescription:

(i) for the S-R, CRUST1, and FWEA18 models with relatively small $h^{\max }$ we take the SAW642AN profile in the range $h=h^{\max }-2900 \mathrm{~km}$.
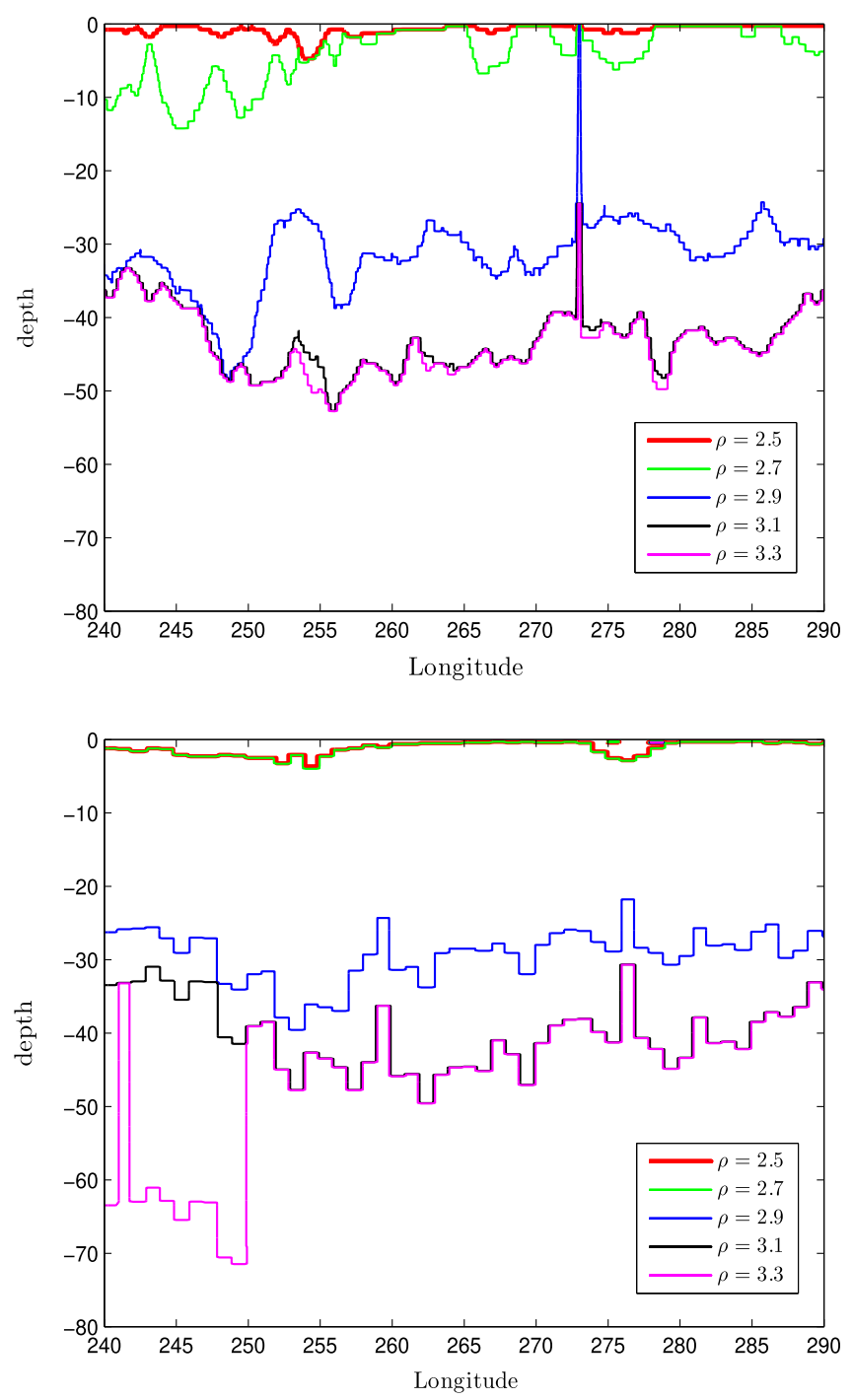

FIG. 2. The depth of layers with several fixed densities beneath the Homestake mine (the latitude $44.35^{\circ}$ ) as function of the azimuthal angle (longitude). The upper panel: Shen-Ritzwoller model, the bottom panel: the CRUST1 model. The black lines show the depth of Moho.

(ii) Below $2900 \mathrm{~km}$ for all the models we use the PREM profile. Recall that PREM-the Preliminary reference Earth model is a one-dimensional model that represents the average (over solid angle) density of the Earth as a function of depth. The depth of Moho in the PREM model equals $h_{\text {Moho }}=24.4 \mathrm{~km}$.

Because of the attenuation effect, possible uncertainties related to these compilations of the profiles do not change the results significantly even for small nadir angles.

(iii) For the purely mantle model SAW642AN above Moho, $h=(0-24) \mathrm{km}$, we take constant density $\rho=\rho_{\mathrm{SAW}}(24 \mathrm{~km})$.

All the models but CRUST1 give the density below sea level. In all simulations, except the case of MICA, 
we consider the surface of Earth as a perfect sphere and take zero density above sea level. The effect of these simplifications is much smaller than sensitivity of all experiments (but MICA) due to restricted statistics. In the case of MICA, we have taken into account the Earth structures above sea level.

In Fig. 2, we present the S-R and CRUST1 density profiles beneath Homestake for fixed latitude $44.35^{\circ}$. Both models provide data for this place down to $80 \mathrm{~km}$. Shown is the depth of layers with a given density as a function of longitude (azimuthal angle). Notice that at the latitude $44^{\circ}$ the $1^{\circ}$ of longitude corresponds to $76 \mathrm{~km}$ at the surface. The black curves show Moho depth, where density jumps approximately from 2.9 to $3.3 \mathrm{~g} / \mathrm{cm}^{3}$.

A few comments are in order.

(1) The surfaces of equal density, and in particular, borders between layers deviate from the spherical form.

(2) There are irregular deviations from the spherical form with typical angular size $(2-5)^{\circ}$ or $(150$ 400) $\mathrm{km}$, which is comparable with the oscillation length. The depth variation $\delta h$ is up to $(5-10) \mathrm{km}$, i.e., up to $30 \%$.

(3) There are narrow spikes of large amplitude and wide regions $\sim 10^{\circ}$, where the depth increases by $30 \%$ with respect to average value.

(4) Two models give rather similar density distributions: the average depths and lengths are similar. At the same time, variations of the S-R and CRUST1 models are not correlated.

In the case of spherical inner structures the nadir angle $\eta_{c}$ at which the neutrino starts to cross a given border between layers with the depth $h$ equals

$$
\sin \eta_{c}=1-\frac{h}{r_{E}}
$$

where $r_{E}=6371 \mathrm{~km}$ is the radius of the Earth. For $\eta<\eta_{c}$ the neutrino crosses this border twice. The neutrino "sees" the mantle for the first time at $\eta_{\text {Moho }}=83.6^{\circ}$ in the S-R model, at $\eta_{\text {Moho }}=83.4^{\circ}$ in the CRUST1 model and at $\eta_{\text {Moho }}=84.9^{\circ}$ in the SAW642AN model on September 23 (where the date fixes the azimuthal angle).

The noticeable difference between the S-R (CRUST1) profile and SAW642AN profile appears above the S-R Moho depth $h>52 \mathrm{~km}$. Below S-R Moho all three models give similar results.

According to Fig. 2 there are deviations of Moho from of ideal sphere of two types:

(i) Relatively small variations of $2^{\circ}-5^{\circ}$ scale which would correspond to (150-400) $\mathrm{km}$ at the DUNE latitude and the size (depth) $\pm(2-5) \mathrm{km}$.

(ii) Long (continental) scale variations of size $50^{\circ}$ with depth $20 \mathrm{~km}$ such that the smallest depth, $h_{\min }=32 \mathrm{~km}$, is close to ocean and the bigger depth $h_{\max }=52 \mathrm{~km}$ is in the center of the continent. This means that the Moho border varies within the shell (we call it Moho shell) restricted by spherical surfaces with depth $32-52 \mathrm{~km}$ and average depth $42 \mathrm{~km}$.

The length of the neutrino trajectory within the Moho shell equals $\approx 2 \sqrt{2 r_{E}\left(h_{\max }-h_{\min }\right)} \approx 710 \mathrm{~km}$, which is 2 times bigger than the oscillation length. According to Eq. (48) borders of the Moho shell are seen from a detector site at $\eta_{\text {min }}=84.2^{\circ}$ and $\eta_{\max }=82.7^{\circ}$. So that for $\eta>\eta_{\text {min }}$ there is no crossings of Moho: in the interval $\eta=$ $\left(\eta_{\min }-\eta_{\max }\right)$ one may expect multiple crossing of Moho and since the horizontal scale of variations of the border is comparable to the oscillation length, parametric effects are expected. However, averaging over azimuthal angle washes out these effects. For $\eta<\eta_{\max }$ the neutrino trajectory crosses the Moho shell twice, and within each crossing, it can be more than one crossing of the Moho border. A substantial effect due to Moho crossings is expected at $\eta \sim 83^{\circ}$.

Below $83^{\circ}$ neutrinos cross the Moho in all the models. For smaller $\eta$ the differences in these models become small.

As an example, in Fig. 3, we show the reconstructed density profiles of three models along the neutrino trajectory which ends at Homestake with $\eta=75^{\circ}$ on September 23. The length of trajectory equals $3295 \mathrm{~km}$. According to Fig. 3 neutrinos cross the Moho border the second time after $3055 \mathrm{~km}$ at a depth of $46 \mathrm{~km}$ in the S-R model. For the CRUST1 model the corresponding numbers are 3121 and $43 \mathrm{~km}$, while for SAW642AN model they equal 3198 and $24 \mathrm{~km}$.

In Fig. 4, similar profiles are shown at the Hida place and or nadir angle $75^{\circ}$.

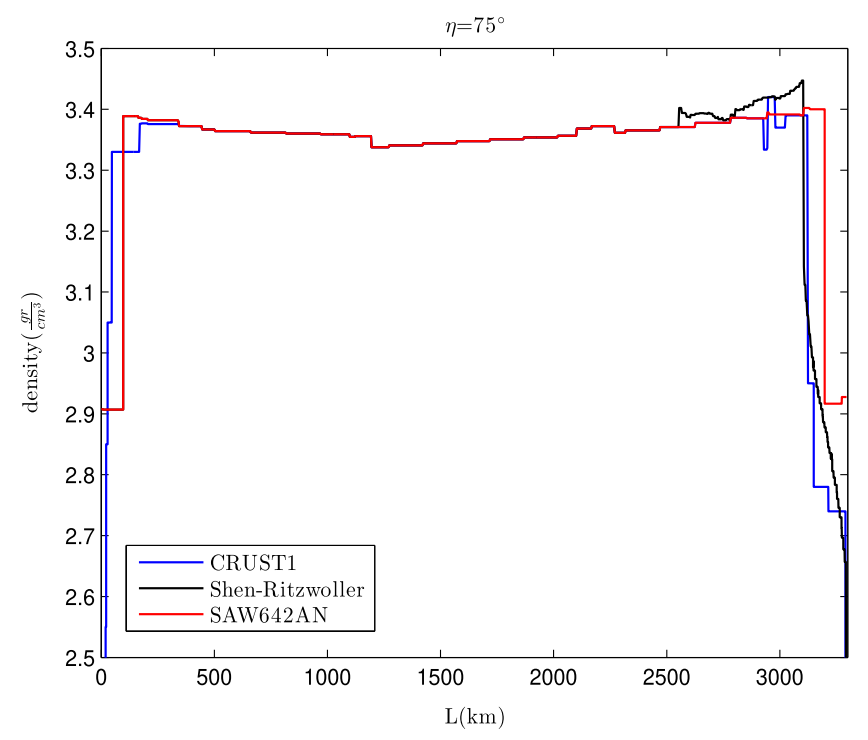

FIG. 3. The density of the Earth along the neutrino trajectory at nadir angle $75^{\circ}$, and detector in Homestake mine as a function of distance from the point of entering the Earth. 


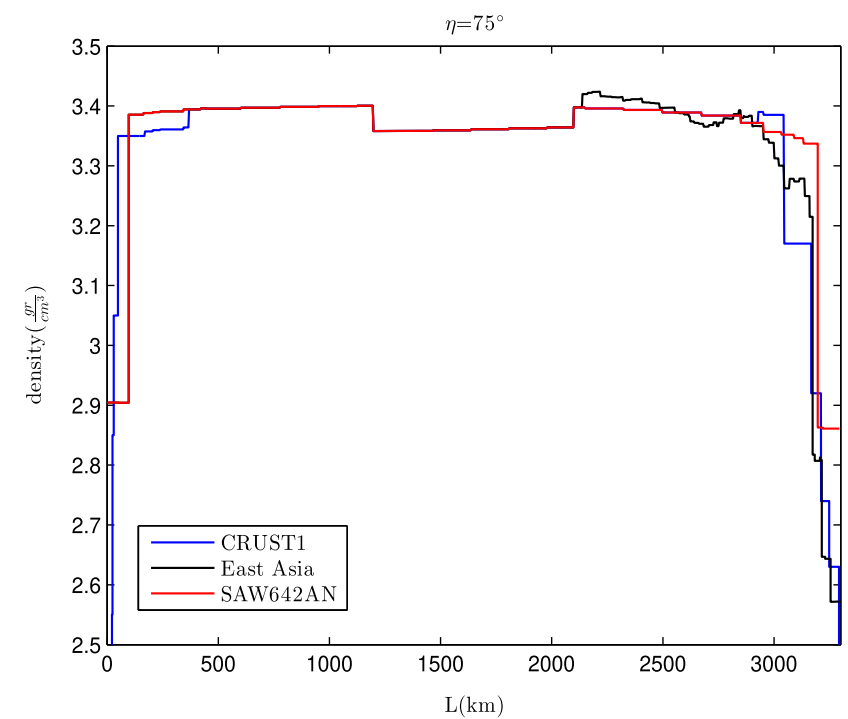

FIG. 4. The same as in Fig. 3 but for the detector located at Hida.

Clearly, the profiles are not symmetric. Moreover, the density decreases to the middle of trajectory, especially for Homestake. This is related to thicker crust in the middle of a continent.

\section{PREDICTIONS FOR FUTURE EXPERIMENTS}

We compute the oscillation probability during a day time $P_{D}(E)$ according to Eq. (2). The rate of events is found using Eq. (31) for $\nu N-$ scattering and Eq. (47) for the $\nu e-$ scattering. The excess of the night event rate was computed using expression in Eq. (32) for the $\nu N-$ scattering and the one in Eq. (44) for the $\nu e-$ scattering. These expressions correspond to $\Delta P$ with neglected $I_{2}$, while the phase was computed keeping the $\epsilon$ correction.

In computations we use the Gaussian functions for $G_{\nu N}\left(E^{r}, E\right)$ and $G_{\nu e}\left(E^{r}, E\right)$ with certain values of the relative widths, $\sigma_{E} / E$. The nadir angle and $A_{N D}\left(\eta, \phi_{a}\right)$ are computed with one minute time intervals during a year. Then we averaged $A_{N D}\left(\eta, \phi_{a}\right)$ over the azimuthal angle $\phi_{a}$.

We performed integration over the energies of produced electrons above certain thresholds. In principle, using narrow energy intervals could improve the energy resolution, and, consequently, sensitivity to remote structures. Notice, however, that with increase of neutrino energy the Earth matter effect increases and the resolution improves. Therefore, due to restricted statistics and the presence of a background the optimal for tomography is integration of events over energy above relatively high threshold. (E.g., for DUNE we use $E^{\text {th }}=11 \mathrm{MeV}$.)

We compute numerically the annual exposures for detectors at Homestake, Hida, and MICA as functions of nadir angle with $\Delta \eta=0.1^{\circ}$ (see Fig. 5). The exposure functions for Homestake is in agreement with that in Ref. [42]. The asymmetry averaged over the year is given

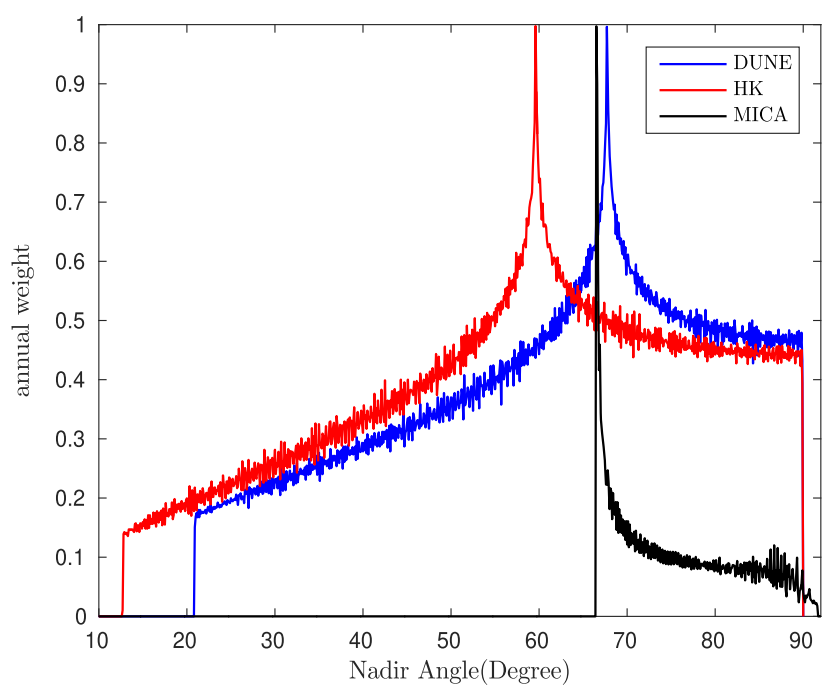

FIG. 5. Annual exposure of the detector as a function of nadir angle for the detectors at Homestake mine, Hida Kamioka, and South pole with the time resolution of $1 \mathrm{~min}$ and nadir angle resolution of $0.1^{\circ}$.

by integration of $A_{D N}$ with the exposure (weight) function $W(\eta)$ over $\eta$ :

$$
\bar{A}_{D N}=\int d \eta W(\eta) A_{D N}(\eta)
$$

We used exposure functions to compute the expected experimental errors for different $\eta$ - intervals. The value $\Delta m_{21}^{2}=7.5 \times 10^{-5} \mathrm{eV}^{2}$ is used unless specially indicated.

\section{A. DUNE}

DUNE is the $40 \mathrm{kt}$ liquid argon TPC which may detect solar neutrinos via the charged current process

$$
\nu_{e}+{ }^{40} \mathrm{Ar} \stackrel{40}{\rightarrow} \mathrm{K}+e^{-} .
$$

For this process we use a generic form of cross section

$$
\sigma_{C C}(E)=A p_{e} E_{e},
$$

where $A$ is a factor irrelevant for the relative excess, $p_{e}$ is the momentum, and $E_{e}=E_{\nu}-\Delta M$ is the energy of the electron with $\Delta M=5.8 \mathrm{MeV}$ being the reaction threshold [42]. Only $9.7 \%$ of ${ }^{8} \mathrm{~B}$ neutrinos have energy $E_{\nu}>11 \mathrm{MeV}$, but due to strong energy dependence in Eq. (50) the corresponding fraction of detected events is 0.9 . Therefore, we use the threshold $11 \mathrm{MeV}$ to achieve higher energy reconstruction. For resolution functions $g_{\nu}$ that enter $G_{\nu N}$ we use $\sigma_{E} / E_{e}=0.1$. With these parameters the width of the generalized resolution function $G_{\nu N}$ turns out to be $\sigma_{E} / E=7 \%$, and consequently, the attenuation length equals $\lambda_{\text {att }}=1800 \mathrm{~km}$ for the average energy $12 \mathrm{MeV}$. The nadir angle at which the length of trajectory 


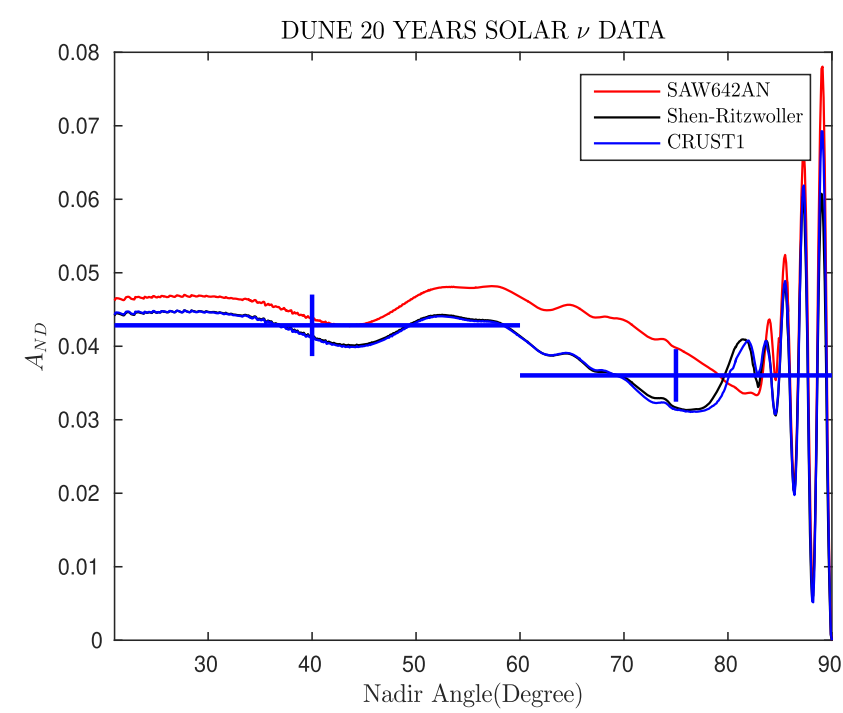

FIG. 6. The night-day asymmetry at DUNE as a function of nadir angle for SAW642AN, Shen-Ritzwoller, and CRUST1 models. The crosses present the expected $1 \sigma$ accuracy of measurements after twenty years of exposure for the CRUST1 model.

$L>\lambda_{\text {att }}$ is $\eta_{\text {att }}=82^{\circ}$. For $\eta<\eta_{\text {att }}$ the Earth structures on the remote part of a neutrino trajectory become invisible.

Results of computations of $A_{N D}(\eta)$ with the S-R, CRUST1, and SAW642AN density profiles are presented in Fig. 6.

Generic features of the $\eta$ dependence of $A_{N D}$ are the following:

(i) Oscillations in crust: Regular oscillatory pattern for $\eta>\eta_{\text {Moho }}$, i.e., $\eta \sim 85^{\circ}-90^{\circ}$ with decreasing depth due to averaging. The third oscillatory peak can be affected by small density jumps in the crust. This quasiregular oscillatory pattern is broken at $\eta_{\text {Moho }}$.

(ii) Moho interference: At $\eta<\eta_{\text {Moho }}$ neutrino trajectory crosses the Moho border twice leading to interference of oscillation waves from two crossings. For some models and values of $\Delta m_{21}^{2}$ the destructive interference of the waves leads to a dip at $\eta_{\text {dip }}$ (for DUNE) which depends on $\eta_{\text {Moho }}$. This can also be interpreted as a parametric suppression of oscillations [42].

(iii) Rise of asymmetry: For $\eta<\eta_{\text {dip }}$, the asymmetry $A_{N D}$ increases with decrease of $\eta$. The increase is due to the fact that for small $\eta$ the section of the neutrino trajectory in the crust becomes much smaller than the oscillation length, and so the effective initial and final densities (averaged over the oscillation length) become larger, being determined by the mantle density.

(iv) In the region $\eta<\eta_{\text {dip }}$ there are bump and another dip due to the effect of density jumps in the mantle at the depths 400 and $670 \mathrm{~km}$.

(v) The core of the Earth $\eta_{\text {core }}=33^{\circ}$ is not seen practically, producing $\sim \epsilon^{2}$ effect at $\eta<\eta_{\text {core }}$.
We find that about $27000 \nu_{e}$ events (49) can be detected annually with $E_{\nu}>11 \mathrm{MeV}$ in the $40 \mathrm{kt}$ fiducial volume according to the CRUST1 model. Our results are comparable to Refs. $[35,42,49]$. The crosses show the expected errors of $A_{N D}(\eta)$ after twenty years of data taking. Statistical errors (computed using the exposure function) are taken into account only and no background was considered. As follows from Fig. 6, the largest difference between SAW642AN and the S-R models as well as SAW642AN and CRUST1, is in the interval $\eta=60^{\circ}-$ $77^{\circ}$ and it originates mainly from different depths of Moho. The difference equals $\Delta A_{N D}(\eta) \sim 0.008$ (15\%) which is about $2 \sigma$ C.L., after 20 years of data taking. The difference between CRUST1 and S-R models is practically negligible. Averaging of $A_{N D}(\eta)$ over $\eta$ leads to $\bar{A}_{N D}=0.040,0.040$, and 0.043, for CRUST1, S-R, and SAW642AN models, respectively, and precision of measurement of $\bar{A}_{N D}$ will be 0.002 .

New models of the Earth density profile have no spherical symmetry, especially in the crust and upper mantle, therefore inclusion of the azimuth angle $\left(\phi_{a}\right)$ dependence of the density profiles in consideration should improve sensitivity to specific models. To illustrate this we divided whole the range of $\phi_{a}$ in to two bins: one bin is to the west and another one to the east from a detector, in addition to two nadir angle bins shown in Fig. 6. Assuming the S-R (or CRUST1) model as the true model, we find that SAW642AN will be disfavored at more than the $2 \sigma$ level, after 20 years of data taking. Integration over the azimuth angle reduces the sensitivity down to $1.6 \sigma$. Because of low statistics in each bin, introduction of more than two $\phi_{a}$ bins will not lead to further improvement of the sensitivity.

The dependence of $A_{N D}$ on $\eta$ in the DUNE experiment computed with the SAW642AN model (red line Fig. 6) is similar to that in Ref. [42] for the PREM model. It has a dip at $\eta_{\text {dip }}=82^{\circ}$ and then an increase of $A_{N D}$ with a decrease of $\eta$. Another dip appears at $\eta=44^{\circ}$. In our present computations (SAW642AN) the dependence $A_{N D}(\eta)$ is smoother than in Ref. [42] below the dip.

\section{B. THEIA}

THEIA is a proposed $100 \mathrm{kT}$ water-based liquid scintillator detector loaded with $1 \%{ }^{7} \mathrm{Li}$ [38]. It will be placed in Homestake. Neutrinos can be detected by the chargedcurrent process

$$
\nu_{e}+{ }^{7} \mathrm{Li} \rightarrow{ }^{7} \mathrm{Be}+e .
$$

The cross section of this process is known with high precision [37,38]. About 17000 events are expected annually with $E_{\nu}>5 \mathrm{MeV}$. In the case of neutrino detection with ${ }^{7} \mathrm{Li}$, we assume $\sigma_{E} / E=12 \%$.

Since THEIA and DUNE are in the same place the results for $A_{N D}(\eta)$ are similar (see Fig. 7, upper panel). The difference between $A_{N D}$ in THEIA and DUNE is due to a 

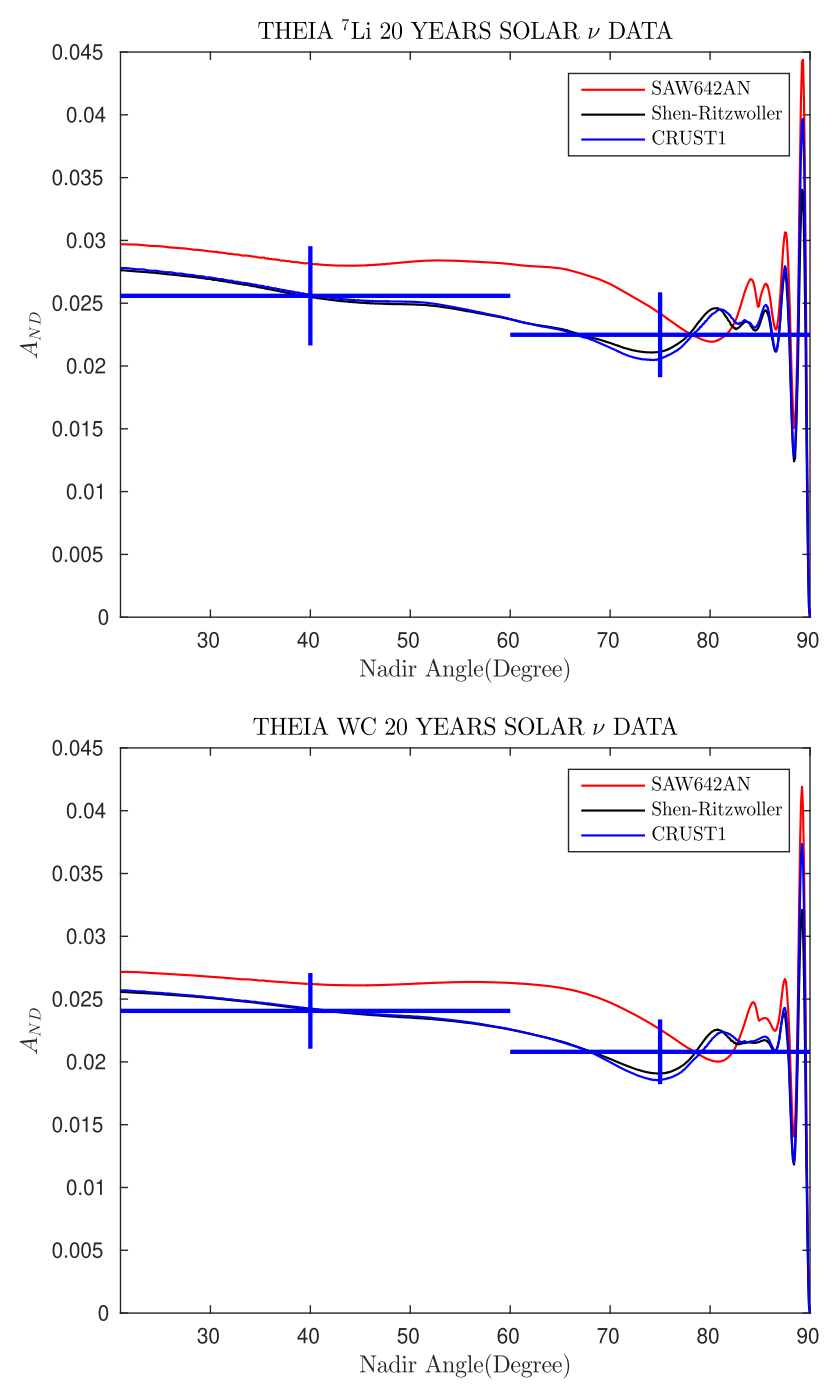

FIG. 7. The same as in Fig. 6, but for THEIA, for detection of neutrinos with ${ }^{7} \mathrm{Li}$ nuclei (upper panel) and elastic scattering on electron (bottom panel).

lower energy threshold in THEIA, which means that effective neutrino energy, and consequently, the oscillation as well as the attenuation lengths are smaller. This, in turn, leads to different interference effects and lower sensitivity to remote structures in THEIA. The difference disappears when the same energy thresholds are taken.

For THEIA the maximal difference of $A_{N D}(\eta)$ computed with S-R and SAW642AN models (and also between CRUST1 and SAW642AN) is about $A_{N D}=0.005$. The difference between the S-R and CRUST1 profile results is much smaller. The values of $A_{N D}$ averaged over $\eta$ with exposure taken into account in the case of ${ }^{7} \mathrm{Li}$ nuclei detection equal to 0.024 (CRUST1), 0.024 (S-R) and 0.027 (SAW642AN).

In THEIA, neutrinos can also be detected via the $\nu-e$ elastic scattering. The asymmetry $A_{N D}$ as a function of $\eta$ (Fig. 7, bottom panel) is similar to that for $\nu^{7} \mathrm{Li}$ detection. Assuming the energy threshold of $6.5 \mathrm{MeV}$ and
$\sigma_{E} / E=0.15$, similar to $\mathrm{HK}$ [36], we find that $A_{N D}$ equals to 0.022 (CRUST1, S-R) and 0.025 (SAW642AN), i.e., slightly smaller than for $\nu^{7} \mathrm{Li}$. Separately, ${ }^{7} \mathrm{Li}-$ and $\nu e-$ detection can discriminate Shen-Ritzwoller (or CRUST1) from SAW642AN at about $1.6 \sigma$ C.L.. Combining the ${ }^{7} \mathrm{Li}$ and $\nu e$ results one can disfavor SAW642AN at more than $2 \sigma$ C.L. Further combining THEIA and DUNE results, SAW642AN will be disfavored at the $2.3 \sigma$ level after $20 \mathrm{y}$ of data taking.

The discrimination between the S-R and CRUST1 models can be improved if for each nadir angle $\eta$ the range of azimuthal angle $\phi_{a}$ is divided into two parts: in the first part $\bar{\rho}_{\mathrm{SR}}>\bar{\rho}_{\mathrm{CRUST} 1}$, and in the second one $\bar{\rho}_{\mathrm{SR}}<\bar{\rho}_{\mathrm{CRUST} 1}$. Then calculating $A_{N D}$ in each of these parts separately and summing up moduli of differences one can avoid averaging.

\section{Hyper-Kamiokande}

Hyper-Kamiokande (HK) will detect the solar neutrinos by the $\nu-e$ elastic scattering with $6.5 \mathrm{MeV}$ threshold [36]. We take $\sigma_{E} / E=15 \%$ as a tentative value. This gives the attenuation length $\lambda_{\text {att }}=700 \mathrm{~km}$ for $E=10 \mathrm{MeV}$.

In Fig. 8, we show the excess of night events computed with FWEA18, SAW642AN, and CRUST1 density profiles. For $d_{\text {Moho }}=33 \mathrm{~km}$ (FWEA18) the nadir angle $\eta_{\text {Moho }}=84.15^{\circ}$, and the length of the trajectory $L=1300 \mathrm{~km}$, so, the remote half of this trajectory will not contribute to the oscillation effect. The dip appears at $\eta_{\text {dip }}=78^{\circ}$ which is intermediate between CRUST1 and SAW64AN.

According to Fig. 8, the maximal difference of $A_{N D}$ in HK computed with FWEA18 and SAW642AN is

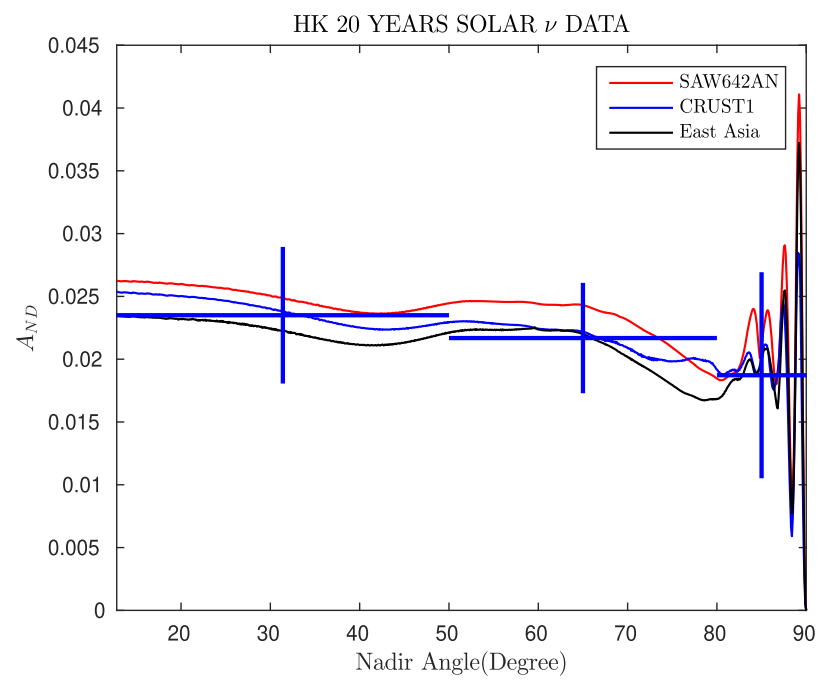

FIG. 8. The day-night asymmetry at Hyper-Kamiokande as a function of the nadir angle for CRUST1, FWEA18, and SAW642AN profiles. The crosses present expected accuracy of measurements after twenty years of exposure taking CRUST1 as the true model. 
$\Delta A_{N D}=0.003$, and appears in the wide range of nadir angles $\eta=10^{\circ}-80^{\circ}$. For the SAW642AN model the $\eta$ dependence in HK is similar to that in the THEIA detector. CRUST1 and FWEA18 have the biggest difference $\Delta A_{N D}=0.004$ in narrow range $\eta=75^{\circ}-80^{\circ}$. Notice that CRUST1 does not produce the dip which is a modeldependent feature. The expected averaged asymmetry $A_{N D}$ in HK equals 0.020 (FWEA18), 0.022 (CRUST1), and 0.024 (SAW642AN). Precision of measurements of $\bar{A}_{N D}$ will be 0.002 after 20 years of exposure with fiducial volume $225 \mathrm{kton}$. We have considered three bins for the nadir angle as demonstrated in Fig. 8. HK will distinguish between the East Asia model and SAW642AN, with $1.5 \sigma$, while the CRUST1 model is recognizable from East Asia and SAW642 with $0.7 \sigma$ and $1.2 \sigma$, respectively, after 20 years of data taking.

The absolute value of asymmetry is substantially smaller than that for DUNE for two reasons: damping due to contribution from NC scattering, which is 0.76 , and difference of averaged energies $E_{\mathrm{HK}} / E_{\mathrm{DUNE}}=0.75$.

\section{MICA}

The Megaton scale Ice Cherenkov Array (MICA) is a proposed detector at the Amundsen-Scott South Pole station [39] in the same place as ICECUBE. The latitude and longitude of MICA are $89.99^{\circ}$ south and $63.45^{\circ}$ west, correspondingly. Crustal structures under Antarctica are not well known due to a lack of seismic data [50], and therefore it is interesting to explore the potential of a solar neutrino detector to determine this structure.

The detection is based on the $\nu-e$ elastic scattering. In our calculations, we took the characteristics of MICA from Ref. [39]: 10 Mton fiducial mass and $10 \mathrm{MeV}$ energy threshold for the kinetic energy of the recoil electron. With these parameters, we find that about $5 \times 10^{5}$ solar $\nu e-$ scattering events are expected per year. For the energy resolution we use $\sigma_{E} / E=15 \%$. We consider the MICA detector at a depth of $2.25 \mathrm{~km}$ below the ice cap (as the Deep Core). The height of ice cap at the location of MICA is $2.7 \mathrm{~km}$ above sea level.

The smallest nadir angle for MICA is $66.5^{\circ}$. About $35 \%$ of the neutrinos have the nadir angle in the interval $66.5^{\circ}-70^{\circ}$. These neutrinos propagate through the Earth with a maximal depth of $500 \mathrm{~km}$. For $\eta=75^{\circ}$ (where the largest difference of $A_{N D}$ from CRUST1 and SAW642AN is expected) neutrinos propagate with a maximal depth of $200 \mathrm{~km}$. Neutrinos reached this angle on May 4 for the first time in a year. According to CRUST1 for $\eta=75^{\circ}$, the depth of Moho is $35 \mathrm{~km}$, with the density jump from 2.9 to $3.4 \mathrm{~g} / \mathrm{cm}^{3}$.

In Fig. 9 we show $A_{N D}(\eta)$ computed with CRUST1 and SAW642AN models. CRUST1 allows taking into account the Earth density above sea level. Since there is no data available for SAW642AN, for this region, we take zero density above sea level. After 20 years of data taking MICA
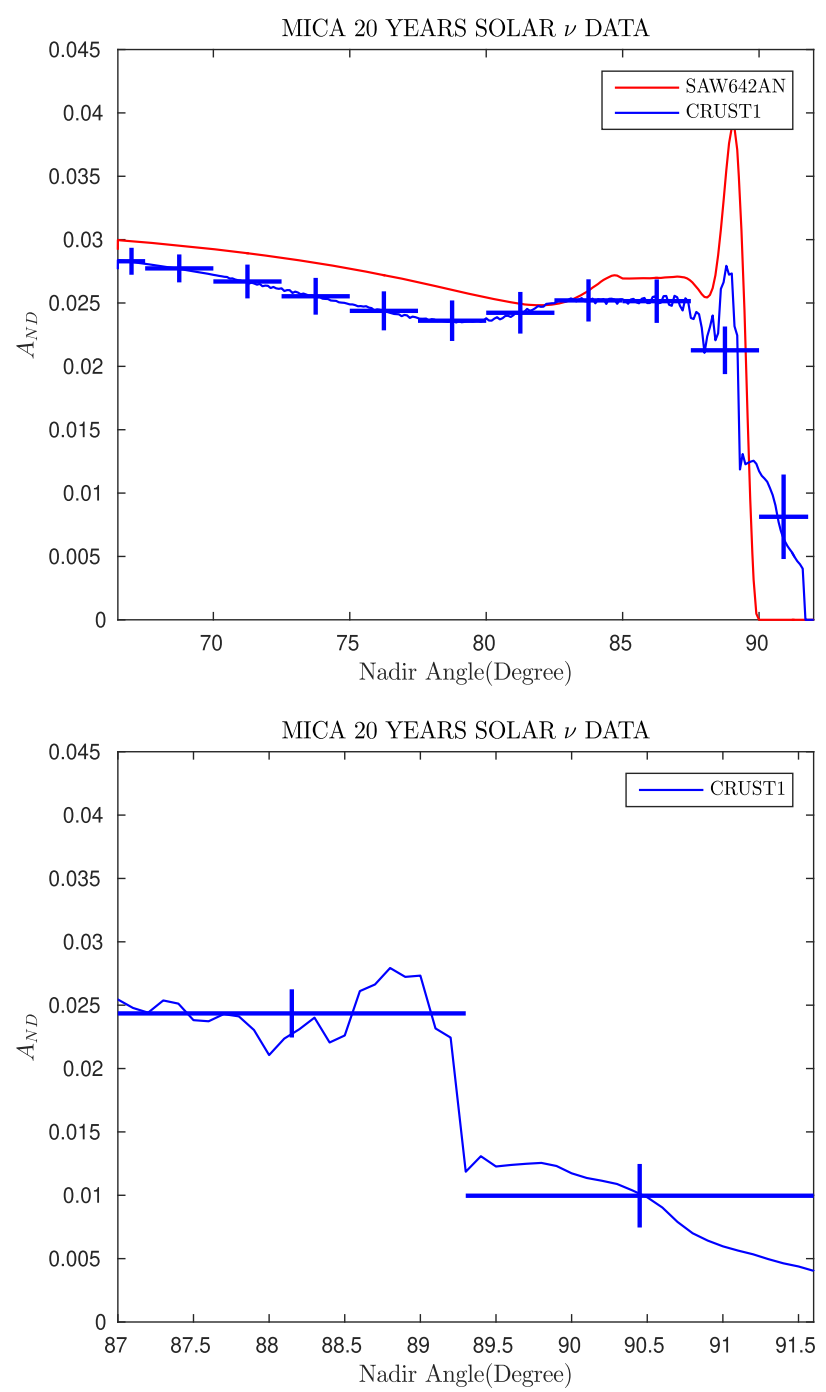

FIG. 9. The night-day asymmetry at MICA as function of $\eta$ for the SAW642AN and CRUST1 models. The crosses present the expected accuracy after $20 \mathrm{yr}$ of exposure and taking the CRUST1 model. Bottom panel shows zoomed part of the upper plot for nadir angles larger than $87^{\circ}$. For $\eta>89.3^{\circ}$, neutrinos cross ice only.

will collect $10^{7}$ solar neutrino events, and it will be sensitive to the ice-soil border. The average value $\bar{A}_{N D}=$ 0.026 in CRUST1 model can be measured with precision 0.00045 . At $\eta>89.3^{\circ}$ neutrinos pass through the ice only, while for smaller $\eta$ they cross the ice-Earth borderline. The SAW642AN model can be excluded with more than $4 \sigma$, assuming that CRUST1 is a true model.

This can be further improved considering the azimuth angle dependence of the density profile. For illustration in addition to 10 nadir angle bins of Fig. 9 we introduced two equal $\phi_{a}$ bins: one to the East and another to the West from the detector. Analysis with 20 bins allows us to exclude SAW642AN at more than $5 \sigma$.

Small ripples in $A_{N D}$ dependence on $\eta$ that appear in the CRUST model (the blue curve in Fig. 9) are real. In this 
model, the surface of the Earth is not spherically symmetric and the density of the Earth above sea level is given. Therefore neutrinos enter the Earth at different heights from sea level, which leads to ripples due to change of the baseline with $\eta$. Such ripples are far from being detected experimentally. The ripples of $A_{N D}$ are absent in the SAW642AN model (the red curve).

Notice that instead of the day, the cycle signal will be measured in MICA during the year. That requires long term stability of the detector.

\section{E. Dependence on $\Delta m_{21}^{2}$; PREM model results}

There is a significant difference in values of $\Delta m_{21}^{2}$ determined by KAMLAND and from a global fit of the solar neutrino data. In this connection we performed computations of $A_{N D}(\eta)$ using the "solar" value $\Delta m_{21}^{2}=$ $5 \times 10^{-5} \mathrm{eV}^{2}$ (Fig. 10). The changes are twofold: the overall asymmetry increases as $1 / \Delta m_{21}^{2}$, i.e., it becomes 1.5 times larger than before. The oscillation and attenuation lengths increase by the same factor 1.5. This, in turn, leads to (i) some change of the interference picture, (ii) enhancement of sensitivity to remote structures, and bigger densities. As a result, at small $\eta$ the enhancement factor of the asymmetry is bigger than 1.5 .

Let us compare results computed for DUNE with the S-R model for two different $\Delta m_{21}^{2}$ (blue line in Fig. 10 and black line in Fig. 6). As expected, for large $\eta$ the amplitude of oscillations of $A_{N D}$ and its average value is 1.6 times larger than those for large $\Delta m_{21}^{2}$ (see Fig. 11). The dip at $77^{\circ}$ disappears. The peak at $50^{\circ}$ is higher by a factor 1.8 . For deeper trajectories (smaller $\eta$ ) the enhancement factor is 1.80-1.85. The reason for this additional increase in the asymmetry above factor 1.5 is that due to larger oscillation

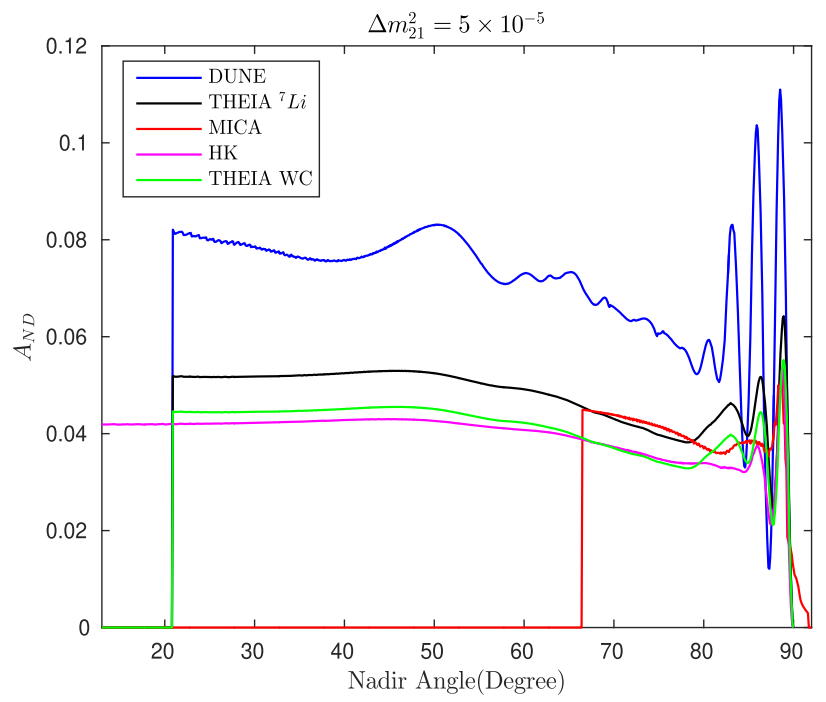

FIG. 10. $A_{N D}$ for $\Delta m_{21}^{2}=5 \times 10^{-5} \mathrm{eV}^{2}$. The $\mathrm{S}$-R model was used for DUNE and THEIA while the CRUST1 model-for HK and MICA.

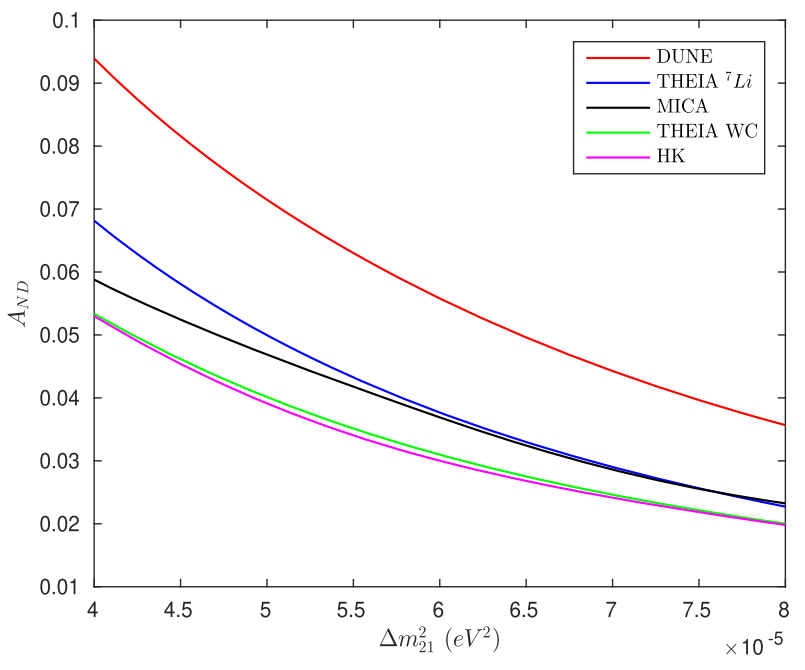

FIG. 11. The averaged over energy $A_{N D}$ as function of $\Delta m_{21}^{2}$ for DUNE and THEIA using the S-R model, and for HK and MICA with CRUST1 model.

length for deep trajectories the effective initial and final densities (averaged over the oscillation length) become larger. For the HK and CRUST1 model the results of $\Delta m_{21}^{2}$ change are similar: For shallow trajectories the asymmetry increases by factor 1.5 , while for deep trajectories (small $\eta$ ) - by factor 2. Dependences of $A_{N D}$ on $\Delta m_{21}^{2}$ for different detectors are shown in Fig. 11.

Notice that using new models of the Earth does not relax the tension between the solar and KamLAND values of $\Delta m_{21}^{2}$. The tension is partially related to the fact that SuperKamiokande found larger D-N asymmetry than is expected for $\Delta m_{21}^{2}$ given by KamLAND. In fact, the situation with SK is similar to that for HK. According to Fig. 12 the averaged $A_{N D}$ computed with CRUST1 model is about 5\% smaller than that with the PREM model. The FWEA18 (East Asia) model gives even smaller $A_{N D}$.

Most of the previous computations were performed with PREM model which has two layers in the crust $(0-15) \mathrm{km}$ and (15-24.4) km and density jumps from 2.6 to $2.9 \mathrm{~g} / \mathrm{cm}^{3}$ at $15 \mathrm{~km}$, and 2.9 to $3.38 \mathrm{~g} / \mathrm{cm}^{3}$ at $24.4 \mathrm{~km}$ (Moho). The $3 \mathrm{~km}$ layer of water is neglected. In Fig. 12 (upper panel) we compare results of PREM (black line) and CRUST1 (blue line) models for DUNE. The difference is mainly related to the depths of Moho: $\eta_{\text {Moho }}=48 \mathrm{~km}$ for CRUST1, which is two times larger than in PREM. Correspondingly, in the CRUST1 model, the dip of $A_{N D}$ is shifted to smaller $\eta$ and for $\eta<\eta_{\text {dip }}$ the asymmetry is smaller. The latter is due to smaller effective density (averaged over the oscillation length) near the detector in CRUST1.

The PREM result is similar to that in Ref. [42]. Less profound oscillatory modulations than in Ref. [42] are related to different treatment of the energy resolution. As we mentioned before, the PREM model result is close to 

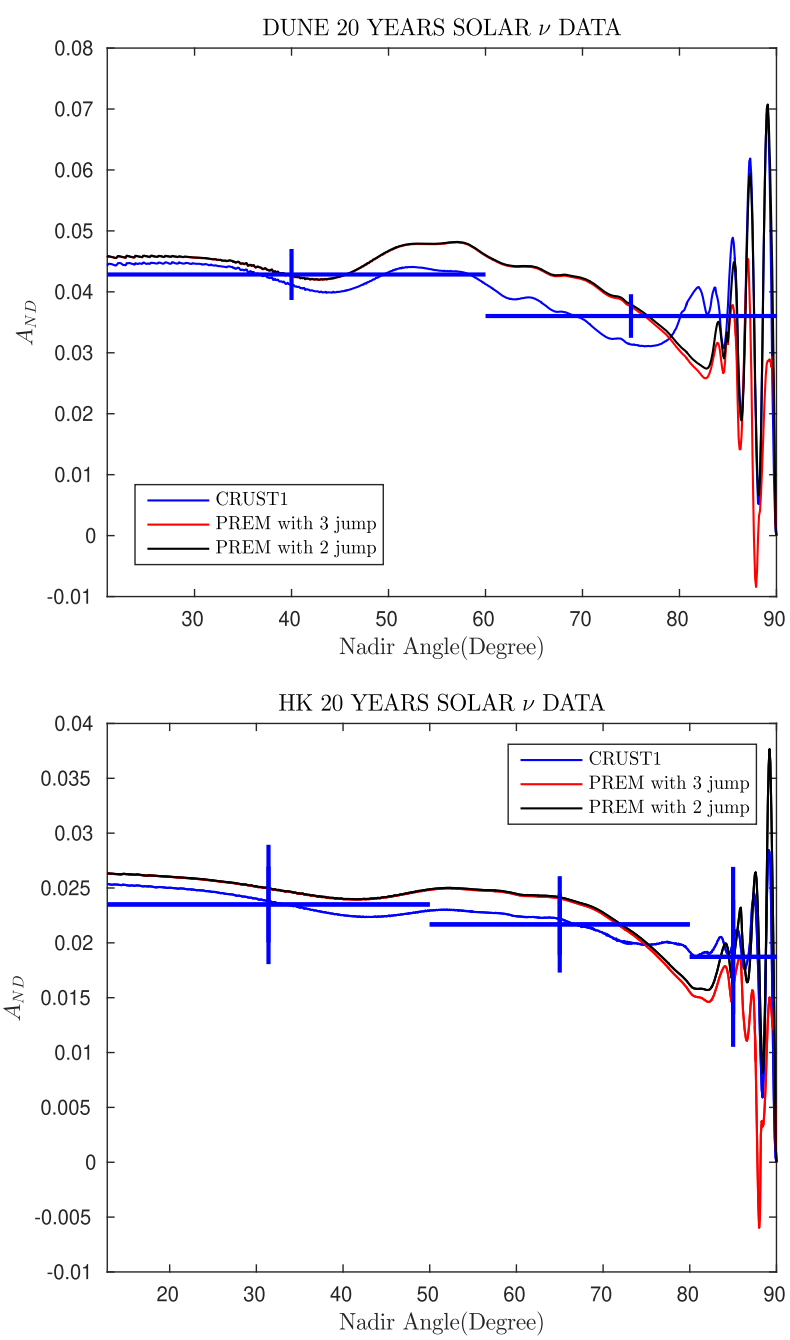

FIG. 12. Comparison of the $\Delta A_{N D}$ dependences on $\eta$ computed using the PREM model with two (black line) and three (red) layers in the crust with $\Delta A_{N D}$ dependence for the CRUST model (blue). Upper panel: for DUNE; bottom panel: for HyperKamiokande.

that of the SAW642AN model which has a similar depth of Moho.

For comparison in Fig. 12 we show also the result for the PREM model with an outer water layer. That would correspond to a detector near the ocean cost. Large differences appear for $\eta>88^{\circ}$, i.e., for trajectories in water: the depth of oscillations and average $\bar{A}_{N D}$ are smaller since they correspond to small water density $1.02 \mathrm{~g} / \mathrm{cm}^{3}$.

Similar situation is for HK Fig. 12 (bottom). According to CRUST1 the dip is absent, $A_{N D}$ is larger in the range $\eta=75^{\circ}-85^{\circ}$, while at $\eta<75^{\circ}$ the asymmetry is $10 \%$ smaller (by 0.002) than for PREM.

The results show that usage of PREM model causes up to $10 \%$ relative systematic error in $A_{N D}$.

Another approach to the oscillation tomography is to use the energy spectrum distortion for fixed direction $\eta$. Inverse problem of reconstruction of the density profile from the energy distortion was considered in Ref. [32]. In particular, the effects of deviation from spherical symmetry were discussed using a toy model.

\section{CONCLUSION}

(1) We performed a detailed study of the Earth matter effects on solar neutrinos using recent 3D models of the Earth. Interesting and non trivial oscillation physics is realized, which is related to complicated density profiles along neutrino trajectories. The day-night asymmetry as a function of the nadir angle has been computed for future experiments DUNE, THEIA, and HyperKamiokande, as well as for possible next-after-next generation experiment MICA. This allows us to assess the feasibility of tomography of the Earth with solar neutrinos.

(2) We estimated corrections to $A_{N D}$ of the order $\sim \epsilon^{2}$. Corrections $\sim \epsilon^{2}$ from $I_{2}$ can be neglected due to an additional small coefficient, while the $\epsilon$ correction to the oscillation phase can be relevant.

(3) We further elaborated on the attenuation effect. The night excess of events and $A_{N D}(\eta)$ are expressed in terms of the matter potential and the generalized energy resolution function which, in turn, determines the attenuation factor. This form is the most appropriate for tomography. We have found that inclusion of energy dependence of the boron neutrino flux and cross section into the resolution function improves the resolution, and therefore sensitivity to remote structures. It is the generalized resolution function that determines sensitivity of oscillation results to the density profile.

Further improvement of the sensitivity can be achieved imposing high enough energy threshold for detected electrons. The gain is twofold: (i) the Earth matter effect increases as $E$; (ii) the attenuation becomes weaker. At the same time loss of statistics is rather moderate.

(4) Using recently elaborated 3D models of the Earth we reconstructed the density, and consequently, potential profiles along neutrino trajectories characterized by coordinates of a detector, nadir, and azimuthal angles. The key feature of the models is the absence of spherical symmetry. Averaging over $\phi_{a}$ leads to dumping of oscillatory modulations.

The key feature of profiles that determines the $A_{N D}(\eta)$ is the depth of Moho (border between crust and mantle). The depth differs substantially in different models, and furthermore, the border substantially deviates from the spherical form.

(5) Difference of results for different models of the Earth at DUNE and THEIA at Homestake is about $10 \%$. After 20 years of DUNE exposure that would correspond to $2 \sigma$ C.L. So, the models cannot be discriminated. A similar conclusion is valid for HK. 
(6) MICA will be sensitive to the ice-soil border. It can discriminate between the CRUST1 and SAW642AN models at $5 \sigma$ C.L. after 20 years of data taking.

(7) With a decrease of $\Delta m_{21}^{2}$ the overall excess increases as $1 / \Delta m_{21}^{2}$. Also $\eta$ dependence changes, which is related to an increase of the oscillation length and therefore decrease of the oscillation phase: for deep trajectories the enhancement with decrease of $\Delta m_{21}^{2}$ is stronger than $1 / \Delta m_{21}^{2}$.

(8) The difference of results obtained for Homestake with S-R and CRUST1 from those of the PREM model, which was used in most of the previous studies, is that the dip in the nadir angle distribution does not appear and for deep trajectories the asymmetry is $10 \%$ lower.

In conclusion, future experiments DUNE, THEIA, and HK will certainly establish the integrated Earth matter effect with high significance. They may observe some generic features of the $\eta$ dependence such as dip and slow increase of the excess with decrease of $\eta$. However, they will not be able to discriminate between recent models. For this, megaton scale experiments like MICA are needed.

\section{ACKNOWLEDGMENTS}

We would like to thank the anonymous referee for useful comments. P. B. would like to thank M. Rajaee, M. Bahraminasr, and M. Maltoni for useful discussions. P. B. received funding from the European Unions Horizon 2020 research and innovation programme under the Marie Skłodowska-Curie Grant Agreement No. 674896 and No. 690575. P. B. is supported by Iran Science Elites Federation Grant No. 11131. P. B. thanks MPIK and IFT for their kind hospitality and support.

\section{APPENDIX: NEUTRINO TRAJECTORY IN THE EARTH}

The Earth can be considered as a sphere with very small (compared to the Earth radius) deviations from the sphere. So, the distance of a given point at the surface from the center of the Earth equals $r_{E}(\theta, \phi)=6371 \mathrm{~km}+H(\theta, \phi)$, where $H(\theta, \phi)$ is the height from sea level of the location. Here, $\theta$ and $\phi$ are the latitude and longitude of the point, respectively. Let us introduce coordinates $x, y$ in the plane perpendicular to the axis of rotation of the Earth and $z$ being along the axis. The axis is tilted by about $\alpha=23.4^{\circ}$ relative to the Earth orbital plane. In these coordinates location of a point on the Earth surface at a given moment of time $t$ is determined by

$$
\begin{aligned}
& x=r_{E}(\theta, \phi) \cos \theta \cos (\phi+\omega t), \\
& y=r_{E}(\theta, \phi) \cos \theta \sin (\phi+\omega t) \cos \alpha-r_{E}(\theta, \phi) \sin \alpha \sin \theta, \\
& z=r_{E}(\theta, \phi) \sin \theta \cos \alpha+r_{E}(\theta, \phi) \cos \theta \sin (\phi+\omega t) \sin \alpha,
\end{aligned}
$$

where $\omega$ is the angular frequency of the Earth rotation.

Location (latitude and longitude) of DUNE and THEIA (Homestake) is $44.35^{\circ}$ of north and $103.75^{\circ}$ of the west. For H-Kamiokande (Hida) we have $36.23^{\circ}$ of north and $137.19^{\circ}$ of the east, and for MICA (Amundsen-Scott South Pole Station): $89.99^{\circ}$ south and $63.45^{\circ}$ west.

In all the cases except for MICA we have considered the Earth surface as a perfect sphere $[H(\theta, \phi)=0]$, and the detectors located at the surface of the Earth. In the case of MICA, we used the CRUST1 model, which allows us taking into account $H(\theta, \phi)$, and the detector is location $2.2 \mathrm{~km}$ below the ice surface.

The coordinates of the Earth in the solar system are

$X=r_{a} \cos \left(\Omega t+\Phi_{0}\right), \quad Y=r_{a} \sin \left(\Omega t+\Phi_{0}\right)$,

where $\Omega$ is $2 \pi /(365.256 \mathrm{~d})$, and $r_{a}=a(1-b \cos \Omega t)$ is the distance between Earth and the Sun. Here $a=1$ is the astronomical unit, and $b=0.0167$ is the eccentricity of the Earth orbit. For the starting point, $t=0$, at the 23rd of September the phase equals $\Phi_{0}=-\frac{\pi}{2}$.

Let $x_{D}$ and $y_{D}$ be the coordinates of the detector and $x, y$, and $z$ are the coordinates of the point at which the neutrino enters the Earth. The neutrino trajectory inside the Earth is determined by solving the following quadratic equation:

$$
x^{2}+y^{2}=r_{D}^{2}, \quad y=m\left(x-x_{D}\right)+y_{D},
$$

where $m \equiv Y / X$ and $r_{D}^{2}=x_{D}^{2}+y_{D}^{2}$. Taking into account tilt $\alpha$, the latitude and longitude of the entering point to the Earth and, consequently, the trajectory of the neutrino inside the Earth as well as the nadir angle are determined.

To perform a precise calculation of the neutrino trajectory for MICA we use the CRUST1 model. In this case, the Earth is not a perfect sphere. Therefore we solved the quadratic equation first with $r_{E}$ that includes $H_{d}$, the depth of the detector from the sea level. In this way, we obtained the entrance point of the neutrinos into the Earth, $\theta_{0}$ and $\phi_{0}$. Then we have solved Eq. (A3) once again with $H\left(\theta_{0}, \phi_{0}\right)$. 
[1] S. P. Mikheyev and A. Yu. Smirnov, Proceedings of the 6th Moriond Workshop on massive Neutrinos in Astrophysics and Particle Physics, Tignes, Savoie, France, edited by O. Fackler and J. T. T. Van (Editions Frontières, Gif sur Yvette, France, 1986), p. 355, https://inspirehep.net/literature/ 243440.

[2] E. D. Carlson, Phys. Rev. D 34, 1454 (1986).

[3] M. Cribier, W. Hampel, J. Rich, and D. Vignaud, Phys. Lett. B 182, 89 (1986).

[4] J. Bouchez, M. Cribier, J. Rich, M. Spiro, D. Vignaud, and W. Hampel, Z. Phys. C 32, 499 (1986).

[5] S. Hiroi, H. Sakuma, T. Yanagida, and M. Yoshimura, Prog. Theor. Phys. 78, 1428 (1987).

[6] A. J. Baltz and J. Weneser, Phys. Rev. D 35, 528 (1987).

[7] A. Dar, A. Mann, Y. Melina, and D. Zajfman, Phys. Rev. D 35, 3607 (1987).

[8] S. P. Mikheyev and A. Yu. Smirnov, Proceeding of 7th Moriond Workshop on Search for New and Exotic Phenomena, Les Arc, Savoie, France, edited by O. Fackler and J. T. T. Van (Editions Frontieres, Gif-sur-Yvette, France, 1987), p. 405.

[9] A. J. Baltz and J. Weneser, Phys. Rev. D 37, 3364 (1988).

[10] A. J. Baltz and J. Weneser, Phys. Rev. D 51, 3960 (1995).

[11] E. Lisi and D. Montanino, Phys. Rev. D 56, 1792 (1997).

[12] Q. Y. Liu, M. Maris, and S. T. Petcov, Phys. Rev. D 56, 599 (1997).

[13] M. Maris and S. T. Petcov, Phys. Rev. D 56, 7444 (1997).

[14] Y. Fukuda et al. (Super-Kamiokande Collaboration), Phys. Rev. Lett. 82, 1810 (1999).

[15] A. Dighe, Q. Y. Liu, and A. Y. Smirnov, arXiv:hep-ph/ 9903329.

[16] A. de Gouvea, A. Friedland, and H. Murayama, J. High Energy Phys. 03 (2001) 009.

[17] J. S. Kim and K. Lee, Comput. Phys. Commun. 135, 176 (2001).

[18] G. L. Fogli, E. Lisi, D. Montanino, and A. Palazzo, Phys. Rev. D 62, 113003 (2000).

[19] C. W. Chiang and L. Wolfenstein, Phys. Rev. D 63, 057303 (2001).

[20] M. C. Gonzalez-Garcia, C. Pena-Garay, and A. Y. Smirnov, Phys. Rev. D 63, 113004 (2001).

[21] M. Maris and S. T. Petcov, Phys. Lett. B 534, 17 (2002).

[22] A. N. Ioannisian and A. Y. Smirnov, arXiv:hep-ph/0201012; M. Blennow, T. Ohlsson, and H. Snellman, Phys. Rev. D 69 , 073006 (2004).

[23] M. B. Smy et al. (Super-Kamiokande Collaboration), Phys. Rev. D 69, 011104 (2004).

[24] A. N. Ioannisian and A. Y. Smirnov, Phys. Rev. Lett. 93, 241801 (2004).

[25] E. K. Akhmedov, M. A. Tortola, and J. W. F. Valle, J. High Energy Phys. 05 (2004) 057.
[26] A. N. Ioannisian, N. A. Kazarian, A. Y. Smirnov, and D. Wyler, Phys. Rev. D 71, 033006 (2005).

[27] J. Hosaka et al. (Super-Kamiokande Collaboration), Phys. Rev. D 73, 112001 (2006).

[28] M. Wurm et al., Phys. Rev. D 83, 032010 (2011).

[29] A. Renshaw et al. (Super-Kamiokande Collaboration), Phys. Rev. Lett. 112, 091805 (2014).

[30] S. S. Aleshin, O. G. Kharlanov, and A. E. Lobanov, Phys. Rev. D 87, 045025 (2013).

[31] O. G. Kharlanov, arXiv:1509.08073.

[32] E. K. Akhmedov, M. A. Tortola, and J. W. F. Valle, J. High Energy Phys. 06 (2005) 053.

[33] A. N. Ioannisian and A. Y. Smirnov, Phys. Rev. D 96, 083009 (2017).

[34] A. M. Dziewonski and D. L. Anderson, Phys. Earth Planet. Inter. 25, 297 (1981).

[35] R. Acciarri et al. (DUNE Collaboration), arXiv:1512.06148.

[36] Hyper-Kamiokande Collaboration, Reports No. KEKPREPRINT-2016-21 and No. ICRR-REPORT-701-2016$1,2016$.

[37] J. R. Alonso et al., arXiv:1409.5864.

[38] M. Askins et al. (Theia Collaboration), Eur. Phys. J. C 80, 416 (2020).

[39] S. Boser, M. Kowalski, L. Schulte, N. L. Strotjohann, and M. Voge, Astropart. Phys. 62, 54 (2015).

[40] J. N. Bahcall, E. Lisi, D. E. Alburger, L. De Braeckeleer, S. J. Freedman, and J. Napolitano, Phys. Rev. C 54, 411 (1996).

[41] D. Adey et al. (Daya Bay Collaboration), Phys. Rev. Lett. 121, 241805 (2018).

[42] A. Ioannisian, A. Smirnov, and D. Wyler, Phys. Rev. D 96, 036005 (2017).

[43] J.S. Monroe and R. Wicander, The Changing Earth: Exploring Geology and Evolution, 5th ed. (Cengage Learning, 2008), p. 216.

[44] B. F. Howell, An Introduction to Seismological Research: History and Development (Cambridge University Press, Cambridge, England, 1990).

[45] W. Shen and M. H. Ritzwoller, J. Geophys. Res. Solid Earth 121, 4306 (2016).

[46] K. Tao, S. P. Grand, and F. N. Niu, Geochem. Geophys. Geosyst. 19, 2732 (2018).

[47] C. Megnin and B. Romanowicz, Geophys. J. Int. 143, 709 (2000).

[48] G. Laske, G. Masters, Z. Ma, and M. Pasyanos, Geophys. Res. Abstr. 15, 2658 (2013), https://meetingorganizer .copernicus.org/EGU2013/EGU2013-2658.pdf.

[49] F. Capozzi, S. W. Li, G. Zhu, and J. F. Beacom, Phys. Rev. Lett. 123, 131803 (2019).

[50] R. Tenzer and M. Bagherbandi, Earth. Sci. Res. J. 17, 712 (2013), http://www.scielo.org.co/scielo.php?script=sci_ arttext\&pid=S1794-61902013000100002. 